\title{
Repurposing combination therapy of Voacamine with Vincristine for down regulation of HIF-1a/FASN co-axis and PHD2 activation in ER+ mammary neoplasia
}

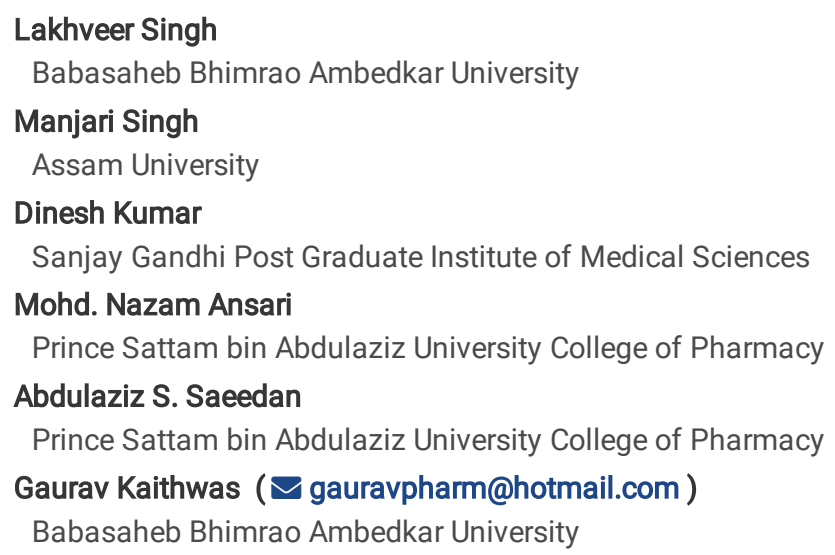

Keywords: Mammary gland carcinoma, Hypoxia inducible factor-1a, Fatty acid synthase, Prolyl hydroxylase-2, Voacamine

Posted Date: August 20th, 2020

DOI: https://doi.org/10.21203/rs.3.rs-61541/v1

License: (c) (i) This work is licensed under a Creative Commons Attribution 4.0 International License. Read Full License 


\section{Abstract \\ Background}

The current study was attempted to inquest the role of combination therapy of Voacamine and Vincristine for the prevention of mammary gland carcinoma through prolyl hydroxylase-2 activation. The prolyl hydroxylase-2 activation leads the downregulation of hypoxia-inducible factor-1a and fatty acid synthase. Over expression of hypoxia inducible factor-1a and fatty acid synthase is previously reported in solid tumor of mammary gland.

\section{Methods}

After screening a battery of natural compounds which were similar to vincristine, vocamine was selected as a possible prolyl hydroxylase-2 activator and justify its activity using 7, 12-Dimethylbenz[a]anthracene induced rat model. The combination therapy was evaluated for cardiac toxicity using hemodynamic profile. The angiogenic markers were evaluated using carmine staining. Monotherapy and combination therapy were also evaluated for liver and kidney toxicity through haematoxylin and eosin staining. The combination therapy also delineated the markers of oxidative stress favorably. Afterwards, the disruption of fatty acids was evaluated using gas chromatography.

\section{Results}

The immunoblotting analysis validated that combination therapy has a potential to switch on the prolyl hydroxylase-2 activity and thus initiate proteolytic degradation of hypoxia-inducible factor- $1 \mathrm{a}$ and its consequence effects. The combination therapy also stimulated programmed cell death (apoptosis) in rapidly dividing cancer cells.

\section{Conclusion}

The present study explores the role of voacamine in activation of prolyl hydroxylase-2 which can decrease over expression of hypoxia-inducible factor-1 $\alpha$ and fatty acid synthase in cells of mammary gland carcinoma.

\section{Background}

The most commonly observed malignancy in females is the mammary gland carcinoma. It is reported that mammary gland cancer makes the one fourth of all the cancers diagnosed every year worldwide [1]. When growth and proliferation of mammary gland tissue is not under control, it divides and forms a lump of cells which transforms into malignant cancer if remain undiagnosed [2]. Despite of much progress in the field of cancer radiation and chemotherapy, treatment and management of mammary gland carcinoma is still very difficult [3]. Moreover, failure of radiation and chemotherapy due to hypoxia in case of solid tumor of mammary gland is another encountered problem [4], Plethora of studies have revealed that hypoxia inducible factor-1a (HIF-1a) is the main component which takes various decisions to attain more hostile environment for proper growth and development of tumor cells even in the scarcity of oxygen [5]. It enhances the expressions of various genes involved in the metabolism of glucose and fatty acid synthesis pathway [6]. In order to synthesize their plasma membrane, rapidly dividing cancer cells require fatty acids, especially polyunsaturated fatty acids (PUFAs) in much larger quantities compared to the normal dividing cells [7]. Therefore, cancer cells adopt an alternative pathway to meet their fatty acid needs. Hence, the mammary gland cancer tissues utilize more glucose and glutamate for their production. So, from above discussion, it was clear that fatty acid plays a very important role in growth and development of mammary gland cancer cells as there is over expression of fatty acid synthase (FASN).

Prolyl hydroxylase-2 (PHD-2) is an important governor of HIF-1a and execute hydroxylation and finally degradation of HIF-1a in presence of oxygen and keep it to minimum level in normal cells [8]. But in case of hypoxic solid tumor, reduced level of oxygen deactivates the PHD-2 and activated HIF-1a. Earlier, our research group conjectured that chemical activation of PHD-2 could curtail the upsurged level of HIF-1a and FASN in mammary gland carcinoma [9-11].

In the present study, we are trying to demonstrate the role of natural compound Voacamine (VOA) alone and in combination with Vincristine (VIN) for mammary gland chemoprevention through activation of PHD-2 and subsequently down regulation of HIF-1a and FASN.

\section{Materials And Methods}

\section{In silico study}

A library of natural compounds was created using Zinc database. The compounds were structural similar with Vincristine (VIN). All the compounds were docked (Autodock 4.2) [12] with PHD-2 protein (PDB ID: 2G19) and about thirteen compounds were found to have good binding energy with

Page 2/20 
PHD-2 protein. After analysis of all the compounds, VOA (Zinc ID169368472) was selected for in vivo study (Fig. 1A).

\section{In vivo study}

\section{Chemicals and Reagents}

VIN (Cytocristin-Cipla) was purchased from the local market. VOA (NCl-3375-85-5) was obtained from National Cancer Institute (NCI) United States as a drug sample. Dimethyl sulfoxide (DMSO) (Merk),, ponceau S (Himedia.ML045), hematoxyline (Himedia, So58), eosin (Himedia,S007), acrylamide (Genetix-1443c196), transfer buffer (Genetix,GX-9411AR), Glycine (Amresco-0167-kg), ammonium persulfate (APS) (LobachemieLB2282a09), RIPA lysis buffer (Amresco,N653), Tetramethylenediamine (TEMED) (Amresco-0761), Tris Ulrapure (Duchefa Biochemie), Sodium Lauryl Sulfate (SLS) (Loba chemieS56971301), DMBA (Sigma Aldrich,57-97-6), Protein assay kit (Amresco, M173), Bovine serum albumin (BSA) (Genetix,PG-2330). All other chemicals used in this study were purchased from Genetix Asia Pvt. Ltd. New Delhi, India and all are of molecular grade.

\section{Experimental animals}

Female albino wistar rats were obtained from CDRI Lucknow after the approval of the protocol (UIP/IAEC/May-2016/06). After procurement, animals were kept in the central animal house in the Department of Pharmaceutical Sciences (BBAU). The animals were kept for $12 \mathrm{~h}$ light/dark cycle at a stable temperature $22-24^{\circ} \mathrm{C}$ ) and humidity. Also, animals had a free access to standard animal feed water ad libitum. All the experiments were performed according to the guidelines laid by CPCSEA, Government of India. After one week, animals were randomly selected (in the weight range 100-150 g) and divided into seven groups containing eight animal in each: Group 1 (normal control receives normal saline $2 \mathrm{ml} / \mathrm{kg}$, p.o.); Group 2 (toxic control receives DMBA $8 \mathrm{mg} / \mathrm{kg}$, i.v.); Group 3 (treatment1(T1) receives DMBA 8 mg/kg, i.v. + VOA 1 mg/kg, s.c.); Group 4 (treatment2 (T2) receives DMBA 8 mg/kg, i.v. + VIN 1 mg/kg, i.v.); Group 5(treatment3 (T3) receives DMBA 8 mg/kg, i.v. + VOA o.5 mg/kg, s.c. + VIN 0.5 mg/kg, i.v) ;Group 6 (treatment4 (T4) receives DMBA 8 mg/kg, i.v. + VOA 1 mg/kg, s.c. + VIN 1 mg/kg, i.v); Group 7 (dummy control receives $3 \%$ DMSO solution s.c.). DMBA was prepared in 3\% DMSO solution and administered once at the beginning of study. Normal saline, $3 \%$ DMSO solution and drugs treatment were administered after 15 days of DMBA injection and continued up to one month at a week interval. The study was continued up to 3 months. At the end of study, blood was collected from retro orbital plexus in order to study the metabolomics profile of control and treatment groups. After blood withdrawal, animals were sacrificed under mild ether anesthesia by cervical dislocation. Abdominal cavity was opened though the median incision. Mammary gland was carefully separated from the skin with the help of sharp scissor and forceps and mounted whole on the slide in order to carry out carmine staining [13]. Liver and kidney was also separated and preserved in the $-20^{\circ} \mathrm{C}$ to be used to assess the toxicity of drug molecules.

\section{Hemodynamic analysis}

For the assessment of cardiac toxicity due to DMBA, hemodynamic profile was performed using AD Instrument. The animals were mounted on the wax trays after anesthetizing by injecting ketamine hydrochloride (50 mg/ $\mathrm{kg}$, i.m.) and diazepam ( $2.5 \mathrm{mg} / \mathrm{kg}$, i.m.). In order to record the ECG signals, dorsal and ventral thorax skin was cleaned and sterilized with spirit and then platinum hook electrodes were fixed on it. These electrodes had connections with the Bio-amplifie (ML-136) and channel power lab (ML-136). Both these instruments work together to convert analogue signals into digital signals (AD Instruments, Australia) which were stored in the hard disk of the system. Offline analysis of saved ECG signals was performed with Lab Chart Pro-8 (AD-Instruments, Australia) [14].

Further, ECG signals were also employed for HRV analysis. Firstly, a manual inspection of recorded signals was carried out in order to accurate detection of R-waves. Afterward, R waves per unit time were plotted in order to calculate the HR. Then, HR was calculated by plotting the number of R-waves per unit time. Finally, Lab Chart Pro-8 (AD Instruments) employed to calculate the time and frequency domain parameters of HRV [15, 16].

\section{Carmine staining}

For mammary gland whole mount analyses, mammary glands tissues were removed from rats and stretched over the glass slides. After drying, slides were placed in the Carnoy's fixative solution [ethanol (60\%), chloroform (30\%) and glacial acetic acid (10\%)] for $2 \mathrm{~h}$ and then washed with decreasing concentrations of ethanol $(90 \%, 70 \%, 35 \%$, and $15 \%)$ for $1 \mathrm{~h}$. The slides were placed in the alum carmine stain ( $1 \mathrm{~g}$ carmine dye and $2.5 \mathrm{~g}$ aluminum potassium sulfate in $500 \mathrm{ml}$ distill water) for 2 days. After 2 days, the Carmine stained slide were taken out and dehydrated with increasing concentration of ethanol (70\%, $95 \%$ and $100 \%)$ and the lipids were removed by immersion in xylene for overnight period of time. After that, slides were examined under the biological miscroscope at $4 \mathrm{X}$ in order to check the presence/absence of alveolar buds (ABs), terminal end duct (TED), terminal end bud (TEB) and lobules (LOs) [17, 18].

\section{Histopathological analysis}

To examine the morphology of mammary gland, liver and kidney; Haemoxyline $(H)$ and Eosin (E) staining was performed. Firstly, tissues were placed in $10 \%$ formaldehyde fixing solution and then buried inside the wax cubes. Using the microtome, $5 \mu \mathrm{m}$ sections were prepared and stained with H\&E. Finally prepared tissue sections were examined under the digital biological microscope (BR-Biochem Life Sciences, N120, New Delhi, India) in order to visualization and imaging of stained tissue. Photographs were taken at 4X and 40X [19]. 


\section{Antioxidant markers}

Frozen mammary gland tissue samples were thawed, precisely weighed and homogenized in $0.15 \mathrm{M} \mathrm{KCl}$. The mixture was centrifuged at $10000 \mathrm{~g}$ for $15 \mathrm{~min}$. Supernatants were collected and kept in ice bath until analysis. The enzymatic assays of catalase, thiobarbituric acid reactive substances (TBARs), superoxide dismutase (SOD), protein carbonyl (PC) and glutathione (GSH) were determined performed according to the method described us earlier [20,21]. The reactivity of the enzymes with tissue samples was determined specrophotometrically (UV-Visible spectrophotometer Agilent Technologies, Carry 60).

\section{Fatty acid methyl ester (FAME) analysis}

Frozen mammary gland tissue was accurately weighed and dissolved in mixture of chloroform: methanol (2:1) in order to make $0.5 \%$ tissue homogenate. The homogenized tissue was further sonicated at $4^{\circ} \mathrm{C}$ up to 5 min and then filtered with Whatman filter paper. Methanol added at last to make up the final volume. In the filtrate, $0.2 \mathrm{ml}$ double distilled water was added to remove the non fatty components. The resultant mixture was left undisturbed for half an hour. After half, an hour, solution was centrifuged at $5000 \mathrm{~g}$ for 5 min. After centrifugation, lower lipid containing layer was conserved and the upper non fatty layer was discarded. In the subsequent step, methyl esters of the lipid sample were prepared by stirring the $0.75 \mathrm{gm}$ of the sample with $2 \mathrm{ml}$ hexane and $0.2 \mathrm{ml}$ methanolic $\mathrm{KOH}(2 \mathrm{~N})$. The resultant mixture was vortexed for $15 \mathrm{~min}$ in order to separate into two layers. The FAME was present in the upper layer. It was carefully separated and used to analyze the lipid profile in control, toxic and treatment groups $[22,23]$.

${ }^{1}$ H NMR study

${ }^{1} \mathrm{H}$ NMR was performed in blood serum samples. To remove precipitates, serum samples were thawed and then centrifuged. For data acquisition, $220 \mu \mathrm{l}$ of supernatant was serum taken in the NMR tubes (Willmad Glass, USA). To make up the final volume $440 \mu$ in the NMR tubes, $220 \mu \mathrm{l}$ NMR buffer solution (sodium phosphate saline of strength $20 \mathrm{~mm}$ of $\mathrm{pH} 7.4$ prepared in D2O) was added. After this, a 2 mm sealed tube called co-axial containing a $0.5 \mathrm{mM}$ solution of 3-trimethylsilysily-(2,2,3,3-d4)-propionic acid (TSP) was inverted in $5 \mathrm{~mm}$ NMR tubes and $150 \mu \mathrm{l}$ of the solution poured into it. It worked as internal reference standard. The prepared samples were analyzed with NMR spectrometer (Bruker NMR spectrometer), $800 \mathrm{MHz}$ ) and raw spectra was obtained as NMR peaks. Also, CPMG (Carr-Purcell-Meiboom-Gill) NMR spectra were recorded for each serum sample by adopting the Brukers standard pulse programme library sequence (cpmgpr1d) with saturation of the water peaks. The spectrum was further processed in Bruker software Topsin-v2.1 (Bruker BioSpin Gmb H, Silberstreinfen 476287 Rheinstetten Germany) and AMIX software in order to carry out spectral binning of the CPMG data. The binned data was then further submitted to MetaboAnalyst, in order to carry out multivariate analysis of metabolomic spectral data. Firstly, Principle Component Analysis (PCA) was performed in order to take initial overview of the metabolites in control, toxic and treatment groupd. Next, data again analyzed with Partial Least Squares Discriminant Analysis (PLS-DA) method to bring out the metabolites responsible for class separation and the class separation among the grouped animals. The data was Pareto scaled for both PCA and PLS-DA and strictly validated for statistical significance. The cross validation of the models was described by the $\mathrm{R}^{2}$ and $\mathrm{Q}^{2}$ parameters and the $\mathrm{p}$-values $\leq 0.05$ calculated with Mann Whitney test for pair wise comparisons) were assumed to be statistical significance $[24,25]$

\section{Immunoblotting}

For protein sample preparations 500 mg mammary gland tissue was weighted and completely homogenized in Radioimmunoprecipitation assay buffer (RIPA lysis buffer) and phenylmethylsulfonyl fluoride (PMSF). The amount of protein in each sample was quantified with Bradford assay. After quantification, proteins were separated on $12.5 \%$ sodium dodecyl sulfate-polyacrylamide gel electrophoresis (SDS-PAGE) gel using the principle of Laemmli. In the subsequent step proteins separated on gel transferred on to the polyvinylidene difluoride (PVDF) membrane (IPVH 00010 Millipore, Bedford, MA USA). Proteins transferred on PVD membrane with Turbo Transfer assembly (Bio Rad) operated at 25 V, 2 A for 15 min at $16 \otimes \mathrm{C}$. The proteins transferred on membrane was blocked for $3 \mathrm{~h}$ with mixture of $5 \% \mathrm{BSA}$ and $5 \%$ not fat skimmed milk prepared in TBST and followed by incubation at $4^{\circ} \mathrm{C}$ with primary antibodies, [ SREBP-1c (SC-13551), HIF-1a (SC-13515), FASN (SC-55580), [(PHD2 (SC-67030)] for overnight period. $\beta$-actin was used as a standard reference. After overnight incubation with primary antibody, PVDF membrane was wiped three times with TBST and then incubated with HRP conjugated secondary antibodies [anti-mouse (SC-31430, Pierce Thermo Scientific, USA), anti-rabbit (SC-2030), anti-goat (SC-2020)] at room temperature for $3 \mathrm{~h}[9,26]$. At the end, membrane was washed one time with TBST and proteins blots were developed and analyzed in ChemiDoc XRS+ (Bio Rad).

\section{Statistical analysis}

The results were analyzed with Graph Pad Prism software (5.02). The values were presented as mean \pm SD and the statistical significance was calculated by one way ANOVA followed by Bonferroni test. The values less than $* p<0.05, * \star p<0.01$, ${ }^{\star \star *} p<0.001$ were considered as statistically significant.

\section{Result}




\section{Effect of drugs and toxicants upon hemodynamic profile}

Hemodynamic profile was measured by ECG (Fig. 1B) and HRV (Table 1). The results were analyzed online by MetaboAnalyst selecting PCA from chemometric analyses. 2D score plot of PCA of NC and treatment groups depicted a very good separation among the groups (Fig. 1C). DMSO treated group was moving away from NC groups which indicates DMSO administration somehow affecting the cardiac activity. DMBA administration also displaced the TC control group. Treatment with monotherpay with VOA (T1) and VIN (T2) further displaced away from the NC. But treatment with combination therapy displaced T3 and T4 even far away from the NC that clearly indicates cardiac toxicity of the drug. To further confirm the cardio toxicity of the drugs, data was again analyzed by Box-Cum Whisker plot (Fig. 1C). A significant difference in R and P amplitude was observed in TC group when compared to the NC and treatment groups. P amplitude increased in all the groups when compared to the NC group with more pronounced effect in VIN treated group. Duration of P wave significantly increased with VOA (T1) but remain same in all the other groups. PR interval increased with high dose monotherapy of VOA, VIN and with high dose combination therapy when compared to the normal control. QT interval was observed to be decreased in DC, combination low and high dose in TC whereas it was observed to be increased with monotherapy (T1 and T2). A raised level of $\mathrm{R}$ amplitude was observed in TC group when compared to all the treatment groups. S amplitude was decreased in DC, T1 and T4 while it was found to be increased with VIN high dose treatment group. T amplitude decreased with DMSO treatment but increased with high dose of monotherapy. A small decrease in heart rate was observed with DMSO administered group but sharp decrease in heart rate was observed with DMBA administration in TC group. Heart rate was even more decrease in all the treatment groups (T1, T2, T3, and T4) with the institution of therapy when compared to the NC, DC and TC. JT interval increased with monotherpapy but opposite trend of RR interval observed in all the treatment groups. Q amplitude was increase with VIN high dose but no change observed with other treatments. T peak was greatly increased with high dose of combination therapy but small increase in T peak also observed with monotherapy.

Table 1: Effect of VOA and VIN on HRV of experimental animals

\section{Time domain}

\begin{tabular}{|c|c|c|c|c|c|c|c|}
\hline Groups & G1-NC & G2-TC & G3-T1 & G4-T2 & G5-T3 & G6-T4 & G7-DC \\
\hline $\begin{array}{l}\text { Average } \\
\text { RR }\end{array}$ & $234.45 \pm 22.98$ & $162.35 \pm 0.070$ & $244.35 \pm 0.07 * \star \star$ & $154.25 \pm 5.30$ & $186.4 \pm 4.94^{\star \star}$ & $147.75 \pm 4.94$ & $195.9 * \star \star$ \\
\hline $\begin{array}{l}\text { Median } \\
\text { RR }\end{array}$ & 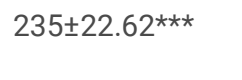 & $163 \pm 0.00$ & $246 \pm 0.00 * * *$ & $154.5 \pm 4.94$ & $185 \pm 1.41 *$ & $148 \pm 1.41$ & 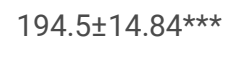 \\
\hline SDRR & $3.806 \pm 1.66$ & $234.45 \pm 22.98^{\star \star \star}$ & $6.465 \pm 0.03$ & $1.40 \pm 0.50 *$ & $9.145 \pm 7.29$ & $1.9 \pm 0.71$ & $5.075 \pm 0.40$ \\
\hline CVRR & $0.016 \pm 0.00 *$ & $0.0465 \pm 0.00$ & $0.024 \pm 0.002$ & $0.0085 \pm 0.00$ ** & $0.0475 \pm 0.038$ & $0.012 \pm 0.004^{*}$ & $0.0255 \pm 0.00$ \\
\hline $\begin{array}{l}\text { Average } \\
\text { Rate }\end{array}$ & $257.25 \pm 25.24^{\star \star \star}$ & $370.4 \pm 0.14$ & $245.7 \pm 0.00 * \star \star$ & $388.3 \pm 11.87$ & $322.9 \pm 7.35^{\star \star \star}$ & $406.15 \pm 3.747 * \star$ & $307.25 \pm 21.99 * * \star$ \\
\hline SD Rate & $4.465 \pm 2.77^{\star * *}$ & $17.635 \pm 1.43$ & $3.158 \pm 0.031^{* \star *}$ & $3.525 \pm 1.08^{* * *}$ & $15.65 \pm 11.74$ & $5.41 \pm 2.149 * *$ & $7.94 \pm 0.48 *$ \\
\hline
\end{tabular}

\section{Frequency domain}




\begin{tabular}{|c|c|c|c|c|c|c|c|}
\hline VLF Band & $28.425 \pm 8.56$ & $30.11 \pm 4.31$ & $54.61 \pm 2.85^{*}$ & $39.63 \pm 17.77$ & 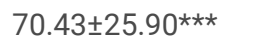 & $30.67 \pm 1.96$ & 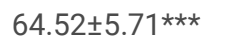 \\
\hline LF & $10.27 \pm 10.13$ & $11.64 \pm 2.22$ & $2.64685 \pm 1.23$ & $7.045 \pm 4.27$ & $11.271 \pm 9.20$ & $5.511 \pm 0.12$ & $5.09 \pm 2.51$ \\
\hline HF & $44.025 \pm 7.67$ & $43.24 \pm 3.37$ & $28.995 \pm 1.025^{\star}$ & $41.265 \pm 6.17$ & $15.9435 \pm 14.51^{\star \star \star}$ & $48.505 \pm 1.80$ & $23.845 \pm 0.37 * \star \star$ \\
\hline LF/HF & $0.2156 \pm 0.19$ & $0.2795 \pm 0.07$ & $0.089 \pm 0.04$ & $0.164 \pm 0.07$ & $0.75 \pm 0.11^{\star \star *}$ & $0.111 \pm 0.001$ & $0.21 \pm 0.09$ \\
\hline
\end{tabular}

All values represent Mean \pm SD. Comparisons were made on the basis of one-way ANOVA followed by Bonferroni test. All groups were compared to the DMBA treated group. Values * $p<0.05,{ }^{* *} p<0.01$ and ${ }^{* *} \mathrm{p}<0.001$ were considered significant.

NC-Normal control (normal saline 3ml/kg,o.p.),TC-Toxic control (DMBA8mg/kg, i.v.),T1-Treatment 1(VOA 1mg/kg, s.c.),T2-Treatment2 (VIN 1mg/kg, i.v.),T3-Treatment3 (VOA 0.5mg/kg, s.c.+ VIN 0.5mg/kg, i.v.), T4-Treatment4 (VOA 1mg/kg, s.c.+ VIN 1mg/kg, i.v.),) DC-Dummy control(DMSO 3\%).

\section{Effect of VOA and VIN upon morphological changes Mammary gland whole mount staining}

Carmine staining is used to analyze the angiogenesis in malignant tumor. Single dose administration of DMBA caused significant increase in angiogenesis marked by increase in alveolar buds (AB) and lobules (LO) in TC group when compared to the NC (Fig. 2A-A1-G1). Administration of monotherapy and combination therapy (both low and high dose) of VOA and VIN inhibited angiogenesis in the experimental animals. Moreover, results shows that VOA alone ( $\mathrm{T} 1$ ) and combination therapy resulted better inhibition of angiogenesis compared to the VIN (T2) treatment characterized by lower number of AB counts and LO.

\section{H\&E staining of sectioned mammary glands}

H\&E staining was performed to view the microscopic architecture of mammary gland tissue of control and toxic treated rats. On DMBA administration, significance damage to the lactiferous duct (LD), adipocytes (AD) along with distorted myoepithelium (ME) and ductal epithelium (DE) was observed in TC group when compared to the NC (Fig. 2A-A2-G2). On initiation of therapy with VOA and VIN (as monotherapy and combination therapy) granted a significant protection to the mammary gland tissue as evidenced by the regeneration of lactiferous duct (LD), fibrous stromal tissue (FST) and adipocytes. A better improvement was observed with the combination therapy comparable to normal. Not any kind of cellular damage was observed in the DMSO (3\%) treated animals. This further gives a clue that DMSO at this concentration has no side effects if used as a solvent.

\section{H\&E staining of liver and kidney tissue}

Results of H\&E staining of rats treated with DMBA caused significant damage to the Bowmans capsule (BC) and glomerules (G) (Fig. 2B). The space between $\mathrm{BC}$ and $\mathrm{G}$ was observed to be increased and structure of proximal convoluted tubules (PCT) and distal convoluted tubule (DCT) observed to be largely distorted in TC when compared to the NC (Fig. 2B: B1, B2). Normal architecture of renal tissue was observed in VOA treated group. But treatment with monotherapy of VIN caused significant damage to BC and G along destruction of microtubules (PCT \& DCT) (Fig. 2B D1, D2). Extensive damage of BC, G, PCT and DCT was observed in treatment groups treated with combination therapy of VOA and VIN (Fig. 2B E1, E2 and F1, F2). Normal architecture of renal tissue was observed in DMSO treated group.

Liver histology of DMBA treated TC showed damage to liver sinusoids and dilation of central vein and resultant disruption of lobular architecture when compared with normal (Fig. 2C:B1 \& B2). Relative small destruction of liver architecture of liver was observed with monotherapy of VOA and VIN (Fig. 2C: C1-C2 \& D1-D2). But extensive damage to lobular structure was observed with both low and dose of combination therapy evidenced by large damage to hepatocytes, sinusoids and dilatation of central vein (Fig. 2C:E1, E2, F1, F2). No damage to liver structure was observed with DMSO administration.

\section{Effect of antioxidant markers upon mammary gland carcinoma}

Single dose administration of DMBA significantly increased the level of TBARs in TC $(0.65 \pm 0.184$ and $119.46 \pm 6.58)$ group when compared to the NC $\left(0.13 \pm 0.032\right.$ and $\left.72.007^{\star * *} \pm 6.64\right)$ group (Table 2). Treatment with monotherapy of VOA and VIN worked well to keep the level of TBARs and

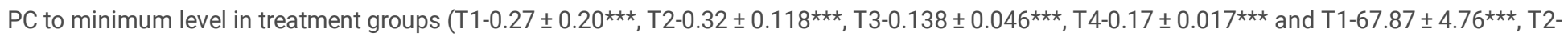
$\left.41.29 \pm 10.66^{\star \star *}, \mathrm{~T} 3-67.38 \pm 10.93^{\star \star *}, \mathrm{~T} 4-44.92 \pm 12.40^{\star \star *}\right)$. Interestingly, treatment with combination low dose therapy provided a better improvement in the level of TBARs whereas in case of PC better restoration of activity was observed with VOA monotherapy and combination low 
dose. GSH, SOD and catalase is a group of enzymes which work together to neutralize the harmful reactive oxygen species (ROS) generated in the body. The level of SOD, catalase and GSH was significantly decreased after DMBA administration in TC (GSH-0.12 \pm 0.005, SOD-0.014 \pm 0.002 , Catalase- $0.29 \pm 0.064$ ) group when compared to the NC (GSH-0.36 \pm 0.002 , SOD- $0.058 \pm 0.006$, catalase-0.72 \pm 0.004$)$ group. It can be observed in the table 1 that level of all three enzymes significantly increased after treatment with monotherapy and combination therapy of VOA and VIN. The combination therapy also imparted a better antioxidant activity.

Table 2

Effect of VOA/VIN on oxidative stress markers

\begin{tabular}{|c|c|c|c|c|c|}
\hline Groups & $\begin{array}{l}\text { TBARS (nM of MDA } \mu \mathrm{g} \text { of } \\
\text { protein) }\end{array}$ & $\begin{array}{l}\text { GSH (mg } \\
\%)\end{array}$ & $\begin{array}{l}\text { SOD(Units of SOD/mg of } \\
\text { protein) }\end{array}$ & $\begin{array}{l}\text { Catalase (nM of H2O2/min/mg } \\
\text { of protein) }\end{array}$ & $\begin{array}{l}\text { Protein carbonyl } \\
\text { (nM/ml unit) }\end{array}$ \\
\hline NC & $0.13 \pm 0.032$ & $\begin{array}{l}0.36 \pm \\
0.002\end{array}$ & $0.058 \pm 0.006$ & $0.72 \pm 0.004$ & $72.007^{\star \star \star} \pm 6.64$ \\
\hline $\mathrm{TC}$ & $0.65 \pm 0.184$ & $\begin{array}{l}0.12 \pm \\
0.005\end{array}$ & $0.014 \pm 0.002$ & $0.29 \pm 0.064$ & $119.46 \pm 6.58$ \\
\hline $\mathrm{T} 1$ & $0.27 \pm 0.20^{\star \star \star}$ & $\begin{array}{l}0.25 \pm \\
0.030\end{array}$ & $0.034^{\star \star \star} \pm 0.002$ & $0.51 \pm 0.123^{\star \star \star}$ & $67.87 \pm 4.76^{\star \star \star}$ \\
\hline $\mathrm{T} 2$ & $0.32 \pm 0.118^{\star \star \star}$ & $\begin{array}{l}0.21 \pm \\
0.002^{\star \star \star}\end{array}$ & $0.041 \pm 0.009^{\star \star \star}$ & $0.45 \pm 0.08^{\star \star \star}$ & $41.29 \pm 10.66^{\star \star \star}$ \\
\hline T3 & $0.138 \pm 0.046^{\star * \star}$ & $\begin{array}{l}0.27 \pm \\
0.001^{\star \star \star}\end{array}$ & $0.039 \pm 0.002^{\star \star \star}$ & $0.53 \pm 0.084^{\star \star \star}$ & $67.38 \pm 10.93^{\star \star \star}$ \\
\hline $\mathrm{T} 4$ & $0.17 \pm 0.017^{\star \star \star}$ & $\begin{array}{l}0.29 \pm \\
0.008^{\star \star \star}\end{array}$ & $0.048 \pm 0.001^{\star \star \star}$ & $0.57 \pm 018^{\star \star \star}$ & $44.92 \pm 12.40^{\star \star \star}$ \\
\hline DC & $0.13 \pm 0.046^{\star * *}$ & $\begin{array}{l}0.39 \pm \\
0.007 \star \star \star\end{array}$ & $0.059 \pm 0.001 * \star \star$ & $0.77 \pm 0.004^{\star \star \star}$ & $75.25 \pm 3.11^{\star \star \star}$ \\
\hline \multicolumn{6}{|c|}{$\begin{array}{l}\text { All values represent mean } \pm \text { SD. Comparisons were made on the basis of one-way ANOVA followed by Bonferroni test. All groups were } \\
\text { compared to the DMBA treated group. Values }{ }^{*} p<0.05,{ }^{*} p<0.01 \text { and } * *{ }^{*} p<0.001 \text { were considered significant. }\end{array}$} \\
\hline \multicolumn{6}{|c|}{ 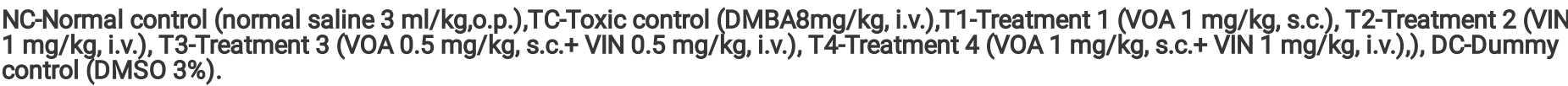 } \\
\hline
\end{tabular}

${ }^{1}$ H-NMR method for serum metabolites profiling

The collected serum samples were analyzed in $1 \mathrm{H}$ NMR to predict change in serum metabolomics and the results are presented in the Fig. 3 (A, $B$ and C). A total of 23 markers namely Glucose, Citrate, Acetate, Lactate, Glycerol, Betaine, Choline, Vakine, Proline, Leucine, Isoleucine, PUFA, Low density lipoproteins (LDL),Very low density lipoproteins (VLDL), Glutamate, Formate, Tyrosine, Fumarate, Phenylalanie, Urea and Histidine were found. A significant raised level of Glucose, Lactate, Actate, Glutamate, Fumarate and PUFAs were observed in TC group when compared to the normal. A significant reduction in aforesaid metabolites observed in the treatment groups with the institution of therapy. Acetate level was reverted back to normal in all the treated groups while level of Lactate was significantly reduced by the combination therapy (favorably with higher dose combination). Monotherapy also worked well in lactate reduction but no significant change in lactate was observed. The level of Glutamate was reduced in T1, T2 and T3 but increased in T4. Glutamine was remain unchanged in T1 but reduced in T2, T3and T4. As far as PUFAs are considered, a linearity pattern of reduction was observed with monotherapy and combination therapy.

\section{Effect of VOA and VIN upon FAME analysis}

FAME analysis of mammary gland tissue of control and treatment groups were analyzed by GC-FID as mentioned in material and method section. A significant change in fatty acid composition of TC animals were observed when compared with NC. Higher levels of PUFAs were observed in TC after DMBA administration. On treatment with VOA and VIN monotherapy as well as with combination therapy, a significant reduction in synthesis of PUFAs was noted (Fig. 4).

\section{Immunoblotting analysis}

Both, combination and monotherapy with VOA and VIN suppressed the expression of HIF-1a, SREBP-1C and FASN. Monotherpy with VOA(T1) $1 \mathrm{mg} / \mathrm{kg}$ ) caused a significant reduction in HIF-1a, FASN and SREBP-1c level when compared to the toxic group while at the same time PHD2 level was observed to be increased. Similarly VIN $1 \mathrm{mg}$ (T2) also provided better inhibition of HIF-1a, FASN and SREBP-1c in comparison to VOA 1 mg group (Fig. 5A). Combination therapy of VOA and VIN both low (T3) and high dose (T4) significantly reduced the expression of HIF-1a, SREBP-1C and FASN in comparison to toxic group. Interestingly, both dose of combination therapy significantly enhanced the PHD2 expression. The results are in consistent with the in silico docking studies. Also, better activation of PHD2 was observed with the high dose (T4) of combination therapy. 
After DMBA administration, a significant change in expression of anti apoptotic (Bcl-XL) and pro-apoptotic (BAD, BAX) proteins were noted in toxic control animals. Also, the increased expression of VDAC, Apaf-1, and Caspase 9 were observed in toxic control. But VOA and VIN therapy imparted significant protection, worked well to re-store the changes (Fig. 5B).

\section{Discussion}

PUFA's are crucial for plasma membrane synthesis in rapidly dividing cancer cells of mammary gland tissue and dietary sources alone are insufficient to accomplish this [27]. Therefore, cancer cells must take over an alternative fatty acid synthesis pathway and HIF-1a helps them to adapt this [28]. Previous studies have reported that HIF-1a acts in a very smart way and modify the tumor microenvironment in such a way that indirectly enhances the fatty acid synthesis, required for the synthesis of plasma membrane and to furnish other purposes in cancer cells [29]. Considering, the role of HIF-1a and FASN, the present study was undertaken to downregulate HIF-1a by activating PHD-2 with natural drugs VOA alone and in combination with VIN.

Cardiac toxicity is very common risk factor in mammary gland carcinoma patient. For the analysis of cardiac toxicity, hemodynamic profile of animals was performed (Fig. 1B, Table 1). Normal ECG and HRV was recorded in case of NC groups when administered vehicle for 3 months. DMSO is a universal solvent for hydrophobic drugs and its cardio toxic affect is well established [30]. DMSO administration decreased the heart rate, S, ST and T interval and increased the RR and P amplitude in DMSO control group. Decrease in HRV after VIN treatment is already reported in previous studies [31] and the same was also reflected in the present study. A large perturbation in ECG and HRV parameters were observed with both low and high dose of combination therapy. Hence, the concomitant administration of VOA with VIN/DMSO would have further exacerbated the cardiotoxic effect (Fig. 1C).

Hypoxia in solid tumors plays important role in angiogenesis which is necessary to accomplish increasing demand of oxygen and other nutrients. Various studies have already reported that increased level of HIF-1a stimulates angiogenesis in tumor cells [32, 33]. In the present study, results of the carmine staining manifest an increase in the $A B$ count and lobules in the toxic control group which clearly depicts the formation of neovascularization (Fig. 2A:A1-G1). But treatment with monotherapy and combination therapy of VOA and VIN reduced the AB count and no of lobules in the experimental animals which indicates suppression of angiogenesis after initiation of therapy. Therapy with VOA and VIN might have regulated HIF-1a pathway to abolish angiogenesis.

Histopatology of mammary gland tissue of carcinogen treated rats have shown extensive damage to the lactiferous duct (LD), adipocytes (AD), ductal epithelium (DE) and myoepithelium as reported in the previous studies [34]. Extensive damage to the micro architecture of mammary tissue like LD, AD, DE and myoepithelium was observed after DMBA administration in TC control (Fig. 2A:A2-G2). A revert effect upon the same was exhibited with VOA and VIN treatment. This indicates VOA and VIN would be working at cellular level to stop tumor progression. Histopathological examination of mammary gland tissue further witnesses the protective action of therapy.

Liver and kidney is the vital and sensitive organ in the body which are responsible for the detoxification and excretion of drugs. Continuous detoxification and excretion of harmful drug molecules specially those which belongs to the anticancer class, can cause harm to these organs and ultimately becomes the reason of nephrotoxicity and hepatotoxicity if dose is not monitored adequately. Most of the anticancer drugs are reported to have varying degree of nephrotoxicity and hepatotoxicity in patients taking them [35]. Nephrotoxicities due to chemotherapy is marked by the necrosis of the proximal convoluted tubule (PCT), distal convoluted tubule (DCT) epithelial cells and injury to the Bowman's capsule, ultimately kidney failure [36]. Histopathological examination of mammary glands of treated rats revealed the same type of damage to the microarchitecture of kidney after DMBA administration (Fig. 2B). Subsequent treatment with VIN monotherapy further exacerbated the renal toxicity as evidenced by the greater damage to the PCT, DCT and Bowmans capsule as its nephorotoxicity is already reported [37]. It would be pertinent to mention that no renal toxicity was noted in rats treated with monotherapy of VOA which confirms its safety in renal failure. Even more damage to kidney was exhibited by both low and high dose of combination therapy marked by the necrosis of the renal tubular epithelial cell, loop of Henle, and glomerulus attributed to the high drug accumulation, either of the two. Since, VOA is already known to have Pgp (efflux pump) inhibitory effect [38], it might have helped intracellular pooling of VIN in the nephron of experimental animals, can be the one possible cause of nephrotoxicity (Fig. 2B).

Liver is the site where most of the drugs undergoes their first pass metabolism (except from those administered through parenteral route [39]. Normal functioning of the liver affected due to continuous exposure to high concentration of cytotoxic substances which then leads to liver failure in some patients. Liver toxicity is marked by dilation of central vein, damages to hepatocytes (He), distorted sinusoids (Sn), and lobules (Lo) which is very well evident in DMBA treated animals (Fig. 2C). Monotherapy of VOA and VIN worked well to stop further damage to liver of experimental animals which documents the liver safety of both drugs at the given dose. But histology of combination therapy treated rat's depicted large damage to the liver organ which again proves the VIN /VOA accumulation, or either of two into the hepatocytes and consequently resultant hepatotoxicity.

Various studies have reported the role of ROS in development of cancer manifested by the increase in TBARs, PC and reduction in the activity of SOD, GSH and Catalase $[40,41]$. Same type of manifestations in the antioxidant markers were also noted in DMBA treated rats. Interestingly, both, 
monotherapy as well as combination therapy effectively restored the TBARs, SOD and other associated antioxidant markers. From this we can expect, restoration of antioxidant activity could be the one possible mechanism behind the anticancer potential of the drug (Table 2 ).

Numerous studies have reported that myriad changes occur in a biological system under diseases condition which can be detected in the biological fluids like blood serum. With this goal serum metabolic study was carried out to extract biomarkers and to understand the interplay between molecular and cellular components (Fig. 3A,B,C) [42, 43]. Previous studies have demonstrated that a hypoxic tumor cells utilize more and more glucose to meet its energy and for biomass accumulation $[44,45]$. Several studies have reported that tumor cells produce high amount of lactate and glutamate that impart benefit to the tumor cells in various ways like fatty acid biosynthesis, immune protection, angiogenesis and invasiveness [46]. The metabolic profile of DMBA treated rats have shown high level of lactate and glutamate and that of PUFAs which is in accordance to the previous studies i.e excess lactate/glutamate is converted into fatty acids. Decreased level of glucose further affirms the above findings. Interestingly, reveres chronological order of above metabolites were observed with VOA and VIN (monotherapy as well as with combination therapy) i.e. decreased level of lacate, glutamate and PUFAs were noted all in the treatment groups. It would be pertinent to mention that monotherapy with VOA and high dose combination therapy of VOA $(1 \mathrm{mg} / \mathrm{kg})$ and $\mathrm{VIN}(1 \mathrm{mg} / \mathrm{kg})$ provided much better fatty acid synthesis inhibitory action compared to the VIN monotherapy and combination low dose. Serum metabolomics analysis of present clearly established a relationship between glycolysis, lactate, glutamate and fatty acid synthesis production (Table 3). 
List of cross analyzed metabolites

\begin{tabular}{|c|c|c|c|c|c|c|c|}
\hline S.No & Metabolites & ppm Values & NC vs TC & $\begin{array}{l}\text { NC vs } \\
\mathrm{T} 1\end{array}$ & NC vs T2 & NC vs T3 & NC vs T4 \\
\hline 1 & Isoleucine & $0.93(t), 1.01(d)$ & $\uparrow$ & $\uparrow$ & $\downarrow$ & $\downarrow$ & $\uparrow$ \\
\hline 2 & Leucine & $0.95(\mathrm{~d}), 0.96(\mathrm{~d})$ & $\downarrow$ & $\uparrow$ & $\downarrow$ & $\downarrow$ & $\uparrow$ \\
\hline 3 & Valine & $0.98(d), 1.04(d)$ & $\uparrow$ & $\uparrow$ & $\downarrow$ & $\downarrow$ & $\uparrow$ \\
\hline 4 & Lactate & $1.33(\mathrm{~d}), 4.12(\mathrm{q})$ & $\uparrow$ & $\uparrow$ & $\uparrow$ & $\uparrow$ & \\
\hline 5 & Alanine & $1.46(\mathrm{~d})$ & $\uparrow$ & $\uparrow$ & $\uparrow$ & $\uparrow$ & $\uparrow$ \\
\hline 6 & Acetate & $1.91(\mathrm{~s})$ & $\uparrow$ & $\uparrow$ & $\uparrow$ & $\uparrow$ & $\downarrow$ \\
\hline 7 & NAG & $2.04(\mathrm{~m})$ & $\uparrow$ & $\downarrow$ & $\downarrow$ & $\downarrow$ & $\downarrow$ \\
\hline 8 & Glutamate & $2.07(\mathrm{~m}), 2.34(\mathrm{~m})$ & $\uparrow$ & $\uparrow$ & $\uparrow$ & $\downarrow$ & $\downarrow$ \\
\hline 9 & Glutamine & $2.11(\mathrm{~m}), 2.43(\mathrm{~m})$ & $\uparrow$ & $\uparrow$ & $\downarrow$ & $\downarrow$ & $\uparrow$ \\
\hline 10 & Citrate & $2.53(d), 2.69(d)$ & $\downarrow$ & $\downarrow$ & $\downarrow$ & $\downarrow$ & $\downarrow$ \\
\hline 11 & Proline & $2.01(\mathrm{~m}), 1.99(\mathrm{~m})$ & $\uparrow$ & $\uparrow$ & $\uparrow$ & $\uparrow$ & $\uparrow$ \\
\hline 12 & Choline & $3.20(\mathrm{~s}), 4.02(\mathrm{~m})$ & $\uparrow$ & $\uparrow$ & $\uparrow$ & $\downarrow$ & $\uparrow$ \\
\hline 13 & GPC & $3.228(\mathrm{~s}), 4.34(\mathrm{~m})$ & $\downarrow$ & $\downarrow$ & $\downarrow$ & $\downarrow$ & $\downarrow$ \\
\hline 14 & Glycine & $3.55(\mathrm{~s})$ & $\downarrow$ & $\downarrow$ & $\downarrow$ & $\downarrow$ & $\downarrow$ \\
\hline 15 & Glycerol & $3.56,3.65$ (d) & $\downarrow$ & $\downarrow$ & $\downarrow$ & $\downarrow$ & $\uparrow$ \\
\hline 16 & Betaine & 3.268 & $\downarrow$ & $\downarrow$ & $\downarrow$ & $\downarrow$ & $\uparrow$ \\
\hline \multirow[t]{5}{*}{17} & Glucose & $3.24(\mathrm{t}), 3.53(\mathrm{q})$ & $\downarrow$ & $\downarrow$ & $\downarrow$ & $\downarrow$ & $\downarrow$ \\
\hline & & $3.49(\mathrm{t}), 3.71(\mathrm{t})$ & & & & & \\
\hline & & $3.40(t), 3.41(t)$ & & & & & \\
\hline & & $3.46(\mathrm{~m}), 3.83(\mathrm{~m})$ & & & & & \\
\hline & & $3.72(q), 3.76(q), 3.84(q), 3.90(q)$ & & & & & \\
\hline 18 & a-Glucose & $4.65(d)$ & $\downarrow$ & $\downarrow$ & $\downarrow$ & $\downarrow$ & $\downarrow$ \\
\hline 19 & $\beta$-Glucose & $5.23(d)$ & $\downarrow$ & $\downarrow$ & $\downarrow$ & $\downarrow$ & $\downarrow$ \\
\hline 20 & Serine & $3.94,3.98(\mathrm{tt})$ & $\uparrow$ & $\downarrow$ & $\downarrow$ & $\uparrow$ & $\downarrow$ \\
\hline 21 & PUFA & 5.29 & $\uparrow$ & $\uparrow$ & $\uparrow$ & $\uparrow$ & $\downarrow$ \\
\hline 22 & Urea & 5.80 & $\uparrow$ & $\uparrow$ & $\uparrow$ & $\uparrow$ & $\uparrow$ \\
\hline 23 & Tyrosine & $6.89(d), 7.18(d)$ & $\uparrow$ & $\uparrow$ & $\uparrow$ & $\uparrow$ & $\uparrow$ \\
\hline \multirow[t]{2}{*}{24} & Phenylalanine & $7.32(\mathrm{~d}), 7.35(\mathrm{t})$ & $\uparrow$ & $\uparrow$ & $\uparrow$ & $\uparrow$ & $\uparrow$ \\
\hline & & $7.42(d)$ & & & & & \\
\hline 25 & Histidine & $7.05(\mathrm{~s}), 7.76(\mathrm{~s})$ & $\uparrow$ & $\uparrow$ & $\uparrow$ & $\uparrow$ & NA \\
\hline
\end{tabular}




\begin{tabular}{|c|c|c|c|c|c|c|c|}
\hline S.No & Metabolites & ppm Values & NC vs TC & $\begin{array}{l}\text { NC vs } \\
\text { T1 }\end{array}$ & NC vs T2 & NC vs T3 & NC vs T4 \\
\hline 26 & Formate & $8.45(\mathrm{~s})$ & $\uparrow$ & $\uparrow$ & $\uparrow$ & $\uparrow$ & $\downarrow$ \\
\hline 27 & LDL/VLDL & $0.88,0.91$ & $\downarrow$ & $\downarrow$ & $\downarrow$ & $\downarrow$ & $\downarrow$ \\
\hline
\end{tabular}

We further performed the FAME analysis of mammary gland tissue to affirm the raised level of fatty acids after DMBA, VOA and VIN treatment. Similar story was also observed in the FAME analysis of mammary gland tissue i.e high level PUFAs were detected toxic control and very low level of PUFAS were noted in the treatment groups (Fig. 4). Results of FAME analysis further evidences that excess lactate was utilized in fatty acid synthesis. No PUFAs like toxic control were observed in FAME analysis of treated animals which clearly indicates VOA and VIN have an inhibitory action on fatty acid synthesis.

Immunoblotting were further performed to investigate the effect of therapy on the proteins of hypoxic and fatty acid synthetic pathway. Several studies have confirmed that the expression of HIF-1a increase in oxygen scarcity which enhances the expression of other genes that indirectly benefits the tumor cells in various ways $[47,48]$. A study conducted by P.Maxwell et al on the wild type (wt) Hepa- 1 cells and derivatives c4,c31 and Rc4 proved that HIF-1a plays crucial role in GLUT one transporter and VEGF [48]. Another study conducted by Wendi Sun et al on HeLa, HCT116 and on cultured human primary epithelial cells showed that HIF-1a increased the level of SREBP-1c and FASN [49]. Intriguingly, similar type of trend was also observed in the present study i.e. the level of HIF-1a, SREBP and FASN expression was up regulated while PHD2 expression was down regulated after DMBA treatment (Fig. 5A). An, opposite effect on the same was observed after the initiation of VOA/VIN therapy. It is already reported that PHD2 is negative regulator of HIF-1a and activation of PHD2 alone can downregulate all its downstream effects [50]. This affirms that VOA and VIN might have activated PHD2 and subsequently the level of HIF-1a along with FASN proteins would have dwindled as it was hypothesized.

Several studies have reported that failure of apoptosis in normal cells is an indication of cancer transformation [51-53]. Decrease in anti-apoptotic proteins (Bcl-XL and $\mathrm{Bcl}-2)$ and increase in pro-apoptotic proteins (BAD,BAX) indicates normal functioning mitochondrial apoptotic pathway [7]. Result of immunoblotting shows increase in expression of Bcl-XL and decrease in BAX and BAD proteins in toxic control after DMBA administration. Furthermore, expression of VDAC, Apaf-1 and caspase 9 were also found elevated which proves the failure of mitochondrial apoptotic pathway. Treatment with monotherapy as well as with combination therapy of VOA and VIN restored the apoptotic pathway in treatment groups which is evidenced by the increase level of cytochrome-c as well (Fig. 5B).

\section{Conclusion}

To conclude, authors would like to submit that combination therapy of VOA and VIN enhances the protein expression of PHD2 and decrease the protein expression of HIF-1a, SREBP-1c and FASN. VOA and VIN persuaded the mitochondrial mediated death pathway to impede the hypoxic microenvironment and curtail de novo fatty acid synthesis (Fig. 6). Our results suggest the possible therapeutic potential of VOA and VIN in combination against mammary gland carcinoma without any untoward effect. The study also validates the need of clinical evaluation of VOA and VIN combination for its future use.

\section{Abbreviations}

PHD2: Prolyl hydroxylase-2, HIF-1a:Hypoxia inducible factor-1a, FASN:Fatty acid synthase, ER+:Estrogen receptor positive, VOA:Voacamine, VIN:Vincristine, NCl:National Cancer Institute, DMSO:Dimethyl sulfoxide, APS:Ammonium per sulfate, TEMED:Tetramethylenediamine, SLS:Sodium lauryl sulfate, BSA:Bovine serum albumin, DMBA:7,12-Dimethyl benzanthracene, ECG:Electrocardiogram, ABs:Albeolar buds, TED:Terminal end duct, TEB:Terminal end bud, FST:Fibrous stromal tissue, Los:Lobules H\&E:Hamatoxyline and Eosin, TBARs:Thiobarbituric acid reactive substances, SOD:superoxide dismutase, PC:protein carbonyl, GSH:glutathione, FAME:Fatty acid methyl ester, NMR:Nuclear magnetic resonance, TSP:3trimethylsilysily-(2,2,3,3-d4)-propionic acid, CPMG:Carr-Purcell-Meiboom-Gill, PCA:Principle Component Analysis, PLS-DA:Partial Least Squares Discriminant Analysis, RIPA lysis buffer:Radioimmunoprecipitation assay buffer, PMSF:phenylmethylsulfonyl fluoride, SDS-PAGE:sodium dodecyl sulfate-polyacrylamide gel electrophoresis, PVDF:polyvinylidene difluoride, SREBP-1c:Sterol regulatory element binding protein-1c, TC:Toxic control, 
NC:Normal control, BC:Bowmans capsule, G:Glomerulus, PCT:Proximal convoluted tubule, DCT:Distal convoluted tubule, PUFA:Poly unsaturated fatty acids, LDL:Low density lipoprotein, VLDL:Very low density lipoproteins, GC-FID:Gas chromatography-Flame ionization detector, LD:Lactiferous duct, AD:Adipocytes, DE:Ductal epithelium, He:Hepatocytes, Sn:Distorted sinusoids, ROS:Reactive oxygen species, GLUT-1:Glucose transporter one, VEGF:Vascular endothelial growth factor

\section{Declarations}

\section{Ethics approval and consent to participate}

Female albino wistar rats were obtained from CDRI Lucknow after the approval of the protocol (UIP/IAEC/May-2016/06). After procurement, animals were kept in the central animal house in the Department of Pharmaceutical Sciences (BBAU). The animals were kept for $12 \mathrm{~h}$ light/dark cycle at a stable temperature $22-24^{\circ} \mathrm{C}$ ) and humidity. Also, animals had a free access to standard animal feed water ad libitum. All the experiments were performed according to the guidelines laid by CPCSEA, Government of India.

\section{Consent for publication}

Not applicable

\section{Availability of data and materials}

The datasets used and analyzed during the current study are available from the corresponding author on reasonable request.

\section{Competing interests}

Authors declare that there is no competing or financial interest.

\section{Funding}

Not applicable

\section{Authors' contributions}

LS carried out the bench work; MS performed the carmine staining and histopathology; DK performed the NMR studies; MNA \& ASS performed the statistical studies and compiled the data; GK perceived the idea, designed and supervised the whole study, prepared and proof read the final manuscript.

\section{Acknowledgement}

Author would like to acknowledge the National Cancer Institute, USA, for providing the drug sample to carry out this research work.

\section{References}

1. Zhang J, Pei R, Pang Z, Ouyang T, Li J, Wang T, Fan Z, Fan T, Lin B, Xie Y. Prevalence and characterization of BRCA1 and BRCA2 germline mutations in Chinese women with familial breast cancer. Breast cancer research treatment. 2012;132(2):421-8.

2. Ciriello G, Gatza ML, Beck AH, Wilkerson MD, Rhie SK, Pastore A, Zhang H, McLellan M, Yau C, Kandoth C. Comprehensive molecular portraits of invasive lobular breast cancer. Cell. 2015;163(2):506-19.

3. Marcotte R, Sayad A, Brown KR, Sanchez-Garcia F, Reimand J, Haider M, Virtanen C, Bradner JE, Bader GD, Mills GB. Functional genomic landscape of human breast cancer drivers, vulnerabilities, and resistance. Cell. 2016;164(1-2):293-309.

4. Guo J, Li L, Guo B, Liu D, Shi J, Wu C, Chen J, Zhang X, Wu J. Mechanisms of resistance to chemotherapy and radiotherapy in hepatocellular carcinoma. TRANSLATIONAL CANCER RESEARCH. 2018;7(3):765-81.

5. Singh M, Devi U, Roy S, Gupta PS, Saraf SA, Kaithwas G. Prolyl hydroxylase mediated inhibition of fatty acid synthase to combat tumor growth in mammary gland carcinoma. Breast Cancer. 2016;23(6):820-9.

6. Singh L, Aldosary S, Saeedan AS, Ansari MN, Kaithwas G. Prolyl hydroxylase 2: a promising target to inhibit hypoxia-induced cellular metabolism in cancer cells. Drug discovery today. 2018;23(11):1873-82.

7. Roy S, Singh M, Rawat A, Kumar D, Kaithwas G. Mitochondrial apoptosis and curtailment of hypoxia-inducible factor-1 a/fatty acid synthase: A dual edge perspective of gamma linolenic acid in ER + mammary gland cancer, Cell Biochemistry and Function (2020). 
8. Ratcliffe P, Koivunen P, Myllyharju J, Ragoussis J, Bovée JV, Batinic-Haberle I, Vinatier C, Trichet V, Robriquet F, Oliver L. Update on hypoxiainducible factors and hydroxylases in oxygen regulatory pathways: from physiology to therapeutics. Hypoxia. 2017;5:11.

9. Singh M, Devi U, Roy S, Gupta PS, Kaithwas G. Chemical activation of prolyl hydroxylase-2 by BBAP-1 down regulates hypoxia inducible factor$1 \mathrm{a}$ and fatty acid synthase for mammary gland chemoprevention. RSC Advances. 2018;8(23):12848-60.

10. Devi U, Singh M, Roy S, Gupta PS, Ansari MN, Saeedan AS, Kaithwas G. Activation of prolyl hydroxylase-2 for stabilization of mitochondrial stress along with simultaneous downregulation of HIF-1a/FASN in ER + breast cancer subtype. Cell Biochem Funct. 2019;37(4):216-27.

11. Devi U, Singh M, Roy S, Tripathi AC, Gupta PS, Saraf SK, Ansari MN, Saeedan AS, Kaithwas G. PHD-2 activation: a novel strategy to control HIF1a and mitochondrial stress to modulate mammary gland pathophysiology in ER + subtype. Naunyn-Schmiedeberg's Archives of Pharmacology. 2019;392(10):1239-56.

12. Ahmed D, Khan MI, Kaithwas G, Roy S, Gautam S, Singh M, Devi U, Yadav R, Rawat J, Saraf S. Molecular docking Analysis and Antidiabetic activity of Rifabutin against STZ-NA induced diabetes in albino wistar rats. Beni-Suef University journal of basic applied sciences. 2017;6(3):269-84.

13. Roy S, Rawat AK, Sammi SR, Devi U, Singh M, Gautam S, Yadav RK, Rawat JK, Singh L, Ansari MN. Alpha-linolenic acid stabilizes HIF-1 a and downregulates FASN to promote mitochondrial apoptosis for mammary gland chemoprevention. Oncotarget. 2017;8(41):70049.

14. Kaithwas G, RAWAT JK, Roy S, Singh M, Gautam S, Yadav R, Ansari MN, Aldossary SA, Saeedan AS. Transcutaneous vagus nerve stimulation regulates the cholinergic anti-inflammatory pathway to counteract 1, 2-dimethyl hydrazine induced colon carcinogenesis in albino wistar rats. Front Pharmacol. 2019;10:353.

15. Mishra RK, Sammi SR, Rawat JK, Roy S, Singh M, Gautam S, Yadav RK, Devi U, Ansari MN, Saeedan AS. Palonosetron attenuates 1, 2-dimethyl hydrazine induced preneoplastic colon damage through downregulating acetylcholinesterase expression and up-regulating synaptic acetylcholine concentration. RSC Advances. 2016;6(46):40527-38.

16. Sammi SR, Rawat JK, Raghav N, Kumar A, Roy S, Singh M, Gautam S, Yadav RK, Devi U, Pandey R. Galantamine attenuates N, N-dimethyl hydrazine induced neoplastic colon damage by inhibiting acetylcholinesterase and bimodal regulation of nicotinic cholinergic neurotransmission. Eur J Pharmacol. 2018;818:174-83.

17. Manral C, Roy S, Singh M, Gautam S, Yadav RK, Rawat JK, Devi U, Ansari MN, Saeedan AS, Kaithwas G. Effect of $\beta$-sitosterol against methyl nitrosourea-induced mammary gland carcinoma in albino rats. BMC Complementary Alternative Medicine. 2016;16(1):1-10.

18. Rani A, Roy S, Singh M, Devi U, Yadav RK, Gautam S, Rawat JK, Ansari MN, Saeedan AS, Prakash A. a-Chymotrypsin regulates free fatty acids and UCHL-1 to ameliorate N-methyl nitrosourea induced mammary gland carcinoma in albino wistar rats. Inflammopharmacology. 2016;24(5):277-86.

19. Roy S, Singh M, Sammi SR, Pandey R, Kaithwas G. ALA-mediated biphasic downregulation of a-7nAchR/HIF-1a along with mitochondrial stress modulation strategy in mammary gland chemoprevention. Journal of cellular physiology. 2019;234(4):4015-29.

20. Rani V, Gautam S, Rawat JK, Singh M, Devi U, Yadav RK, Roy S, Kaithwas G. Effects of minocycline and doxycycline against terbutaline induced early postnatal autistic changes in albino rats. Physiol Behav. 2018;183:49-56.

21. Gautam S, Singh P, Singh M, Roy S, Rawat JK, Yadav RK, Devi U, Gupta PS, Saraf SA, Kaithwas G. Rifaximin, a pregnane X receptor (PXR) activator regulates apoptosis in a murine model of breast cancer. RSC Advances. 2018;8(7):3512-21.

22. Singh M, Kasna S, Roy S, Aldosary S, Saeedan AS, Ansari MN, Kaithwas G. Repurposing mechanistic insight of PDE-5 inhibitor in cancer chemoprevention through mitochondrial-oxidative stress intervention and blockade of DuCLOX signalling. BMC Cancer. 2019;19(1):1-15.

23. Yadav S, Tiwari V, Singh M, Yadav RK, Roy S, Devi U, Gautam S, Rawat JK, Ansari MN, Saeedan AS. Comparative efficacy of alpha-linolenic acid and gamma-linolenic acid to attenuate valproic acid-induced autism-like features. Journal of physiology biochemistry. 2017;73(2):18798.

24. Roy S, Singh M, Rawat A, Devi U, Gautam S, Yadav RK, Rawat JK, Ansari MN, Saeedan AS, Kumar D. GLA supplementation regulates PHD2 mediated hypoxia and mitochondrial apoptosis in DMBA induced mammary gland carcinoma. Int J Biochem Cell Biol. 2018;96:51-62.

25. Gautam S, Rawat AK, Sammi SR, Roy S, Singh M, Devi U, Yadav RK, Singh L, Rawat JK, Ansari MN. DuCLOX-2/5 inhibition attenuates inflammatory response and induces mitochondrial apoptosis for mammary gland chemoprevention. Front Pharmacol. $2018 ; 9: 314$.

26. Yadav RK, Singh M, Roy S, Gautam S, Rawat JK, Singh L, Ansari MN, Saeedan AS, Kaithwas G. Evaluation of a-linolenic acid-based intramammary nanosuspension for treatment of subclinical mastitis. J Dairy Sci. 2020;103(3):2701-6.

27. Yadav RK, Singh M, Roy S, Ansari MN, Saeedan AS, Kaithwas G. Modulation of oxidative stress response by flaxseed oil: Role of lipid peroxidation and underlying mechanisms. Prostaglandins Other Lipid mediat. 2018;135:21-6.

28. Bensaad K, Favaro E, Lewis CA, Peck B, Lord S, Collins JM, Pinnick KE, Wigfield S, Buffa FM, Li J-L. Fatty acid uptake and lipid storage induced by HIF-1 a contribute to cell growth and survival after hypoxia-reoxygenation. Cell reports. 2014;9(1):349-65.

29. Furuta E, Pai SK, Zhan R, Bandyopadhyay S, Watabe M, Mo Y-Y, Hirota S, Hosobe S, Tsukada T, Miura K. Fatty acid synthase gene is upregulated by hypoxia via activation of Akt and sterol regulatory element binding protein-1. Can Res. 2008;68(4):1003-11. 
30. Kramer K, Van SA, Grimbergen JA, Van WderV, Bast A. Effect of dimethyl sulfoxide (DMSO) on the electrocardiogram (ECG) in freely moving male Balb/c mice. Gen Pharmacol. 1995;26(6):1403-7.

31. Ilgin S, Kilic V, Baysal M, Aydogan-Kilic G, Ucarcan S, Dermenci B, Atli O. Evidence for cardiotoxicity associated with sertraline in rats. Toxicology research. 2018;7(5):817-25.

32. Veschini L, Belloni D, Foglieni C, Cangi MG, Ferrarini M, Caligaris-Cappio F, Ferrero E. Hypoxia-inducible transcription factor-1 alpha determines sensitivity of endothelial cells to the proteosome inhibitor bortezomib. Blood. 2007;109(6):2565-70.

33. Sonveaux P, Copetti T, De Saedeleer CJ, Végran F, Verrax J, Kennedy KM, Moon EJ, Dhup S, Danhier P, Frérart F. Targeting the lactate transporter MCT1 in endothelial cells inhibits lactate-induced HIF-1 activation and tumor angiogenesis, PLoS One 7(3) (2012).

34. Hoshino A, Yee CJ, Campbell M, Woltjer RL, Townsend RL, Van Der Meer R, Shyr Y, Holt JT, Moses HL, Jensen RA. Effects of BRCA1 transgene expression on murine mammary gland development and mutagen-induced mammary neoplasia. Int J Biol Sci. 2007;3(5):281.

35. Małyszko J, Kozłowska K, Kozłowski L, Małyszko J. Nephrotoxicity of anticancer treatment. Nephrology Dialysis Transplantation. 2017;32(6):924-36.

36. Perazella MA. Onco-nephrology: renal toxicities of chemotherapeutic agents. Clin J Am Soc Nephrol. 2012;7(10):1713-21.

37. Aitchison R, Reilly I, Morgan A, Russell N. Vincristine, adriamycin and high dose steroids in myeloma complicated by renal failure. British journal of cancer. 1990;61(5):765.

38. Condello M, Cosentino D, Corinti S, Di Felice G, Multari G, Gallo FR, Arancia G, Meschini S. Voacamine modulates the sensitivity to doxorubicin of resistant osteosarcoma and melanoma cells and does not induce toxicity in normal fibroblasts. Journal of natural products. 2014;77(4):855-62.

39. Maor Y, Malnick S. Liver injury induced by anticancer chemotherapy and radiation therapy, International journal of hepatology 2013 (2013).

40. Tiwari V, Singh M, Rawat JK, Devi U, Yadav RK, Roy S, Gautam S, Saraf SA, Kumar V, Ansari N. Redefining the role of peripheral LPS as a neuroinflammatory agent and evaluating the role of hydrogen sulphide through metformin intervention. Inflammopharmacology. 2016;24(5):253-64.

41. Al-Saeedan AS, Gautam V, Ansari MN, Singh M, Yadav RK, Rawat JK, Devi U, Gautam S, Roy S, Kaithwas G. Revisiting the systemic lipopolysaccharide mediated neuroinflammation: Appraising the effect of L-cysteine mediated hydrogen sulphide on it. Saudi pharmaceutical journal. 2018;26(4):520-7.

42. Shang L, Wang M. Molecular alterations and clinical relevance in esophageal squamous cell carcinoma. Frontiers of medicine. 2013;7(4):40110.

43. Hu J-M, Sun H-T. Serum proton NMR metabolomics analysis of human lung cancer following microwave ablation. Radiat Oncol. 2018;13(1):40.

44. Sormendi S, Wielockx B. Hypoxia pathway proteins as central mediators of metabolism in the tumor cells and their microenvironment. Frontiers in immunology. 2018;9:40.

45. Netea-Maier RT, Smit JW, Netea MG. Metabolic changes in tumor cells and tumor-associated macrophages: a mutual relationship. Cancer letters. 2018;413:102-9.

46. Palazon A, Tyrakis PA, Macias D, Veliça P, Rundqvist H, Fitzpatrick S, Vojnovic N, Phan AT, Loman N, Hedenfalk I. An HIF-1a/VEGF-A axis in cytotoxic T cells regulates tumor progression, Cancer cell 32(5) (2017) 669-83. e5.

47. Dales JP, Garcia S, Meunier-Carpentier S, Andrac-Meyer L, Haddad O, Lavaut MN, Allasia C, Bonnier P, Charpin C. Overexpression of hypoxiainducible factor HIF-1a predicts early relapse in breast cancer: Retrospective study in a series of 745 patients. International journal of cancer. 2005;116(5):734-9.

48. Maxwell P, Dachs G, Gleadle J, Nicholls L, Harris A, Stratford I, Hankinson O, Pugh C, Ratcliffe P, Hypoxia-inducible factor-1 modulates gene expression in solid tumors and influences both angiogenesis and tumor growth, Proceedings of the National Academy of Sciences 94(15) (1997) 8104-8109.

49. Sun W, Kato H, Kitajima S, Lee KL, Gradin K, Okamoto T, Poellinger L. Interaction between von Hippel-Lindau protein and fatty acid synthase modulates hypoxia target gene expression. Scientific reports. 2017;7(1):1-11.

50. Chen T, Zhou Q, Tang H, Bozkanat M, Yuan JXJ, Raj JU, Zhou G. miR-17/20 Controls Prolyl Hydroxylase 2 (PHD 2)/Hypoxia-Inducible Factor 1 (HIF 1) to Regulate Pulmonary Artery Smooth Muscle Cell Proliferation. Journal of the American Heart Association. 2016;5(12):e004510.

51. Besra SE, Ray M, Dey S, Roy S, Deb N. Apoptogenic activity of secretion extract of Bellamya Bengalensis $\mathrm{f}$. annandalei via mitochondrial mediated caspase cascade on human leukemic cell lines. International Journal of Pharmaceutical Science Review Research. 2013;20:146-52.

52. Dey S, Roy S, Deb N, Sen KK, Besra SE. Anti-carcinogenic activity of Ruellia tuberosa L.(Acanthaceae) leaf extract on hepatoma cell line \& increased superoxide dismutase activity on macrophage cell lysate. Int J Pharm Pharm Sci. 2013;5(3):854-61.

53. Roy S, Deb N, Basu S, Besra SE. Apoptotic activity of ethanolic extract of Moringa oleifera root bark on human myeloid leukemia cells via activation of caspase cascade. World J Pharm Sci. 2014;3:1138-56. 
Figures

Figure 1A

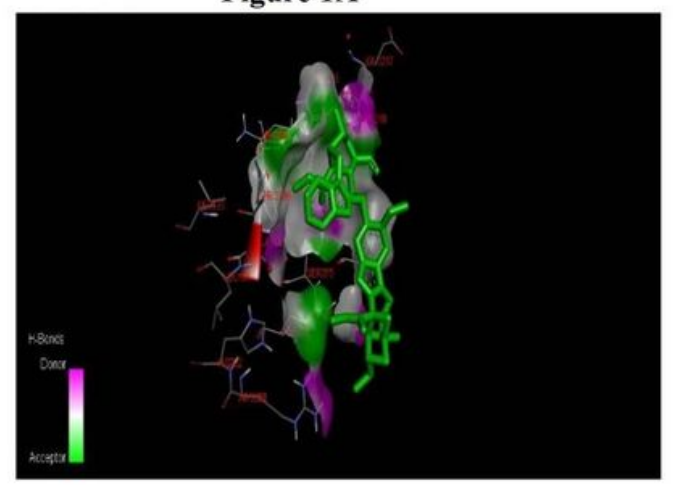

Figure 1B
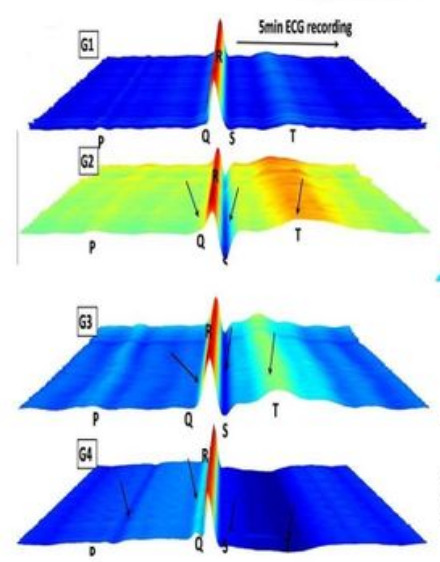
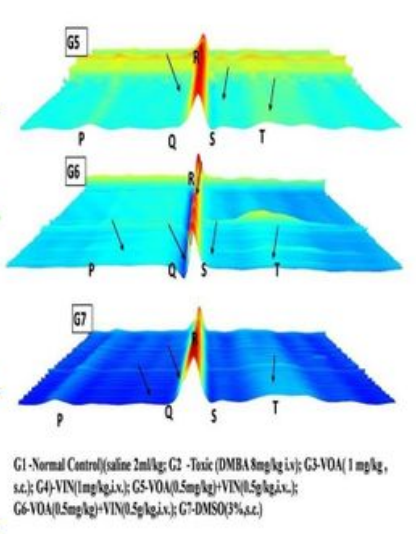

Figure 1C
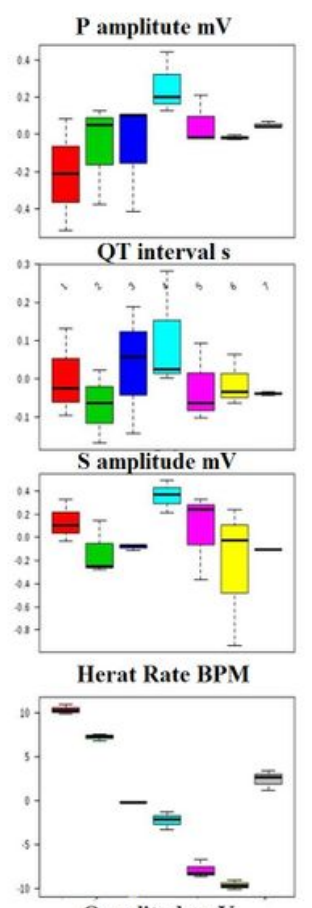

Q amlitude $\mathrm{mV}$

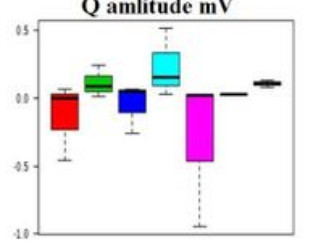

$P$ duration s

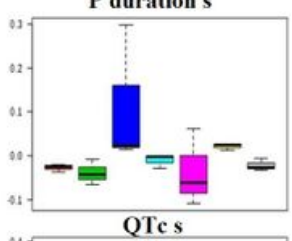

QTes

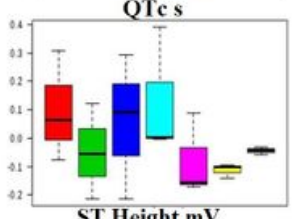

ST Height $\mathrm{mV}$

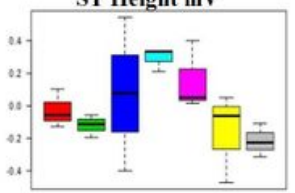

JT Interval s

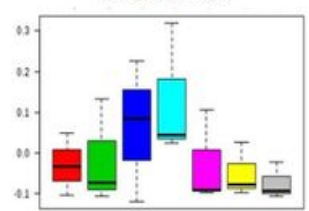

T peak trend interval $s$

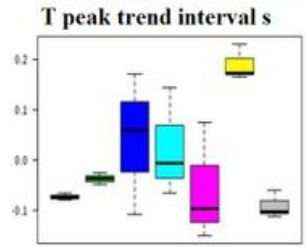

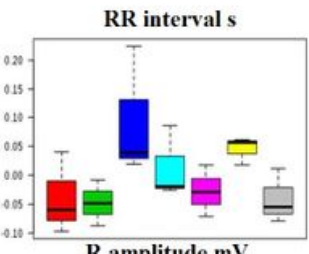
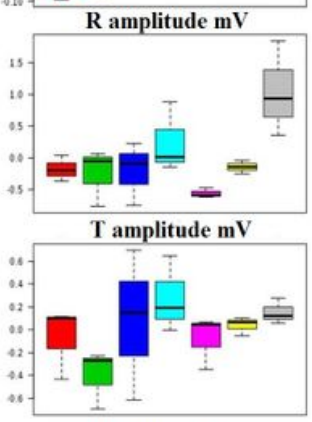

RR Interval s

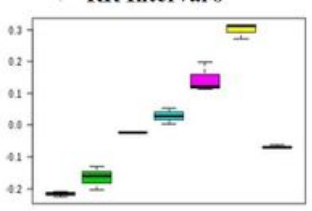

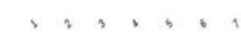

\section{Figure 1}

Figure1A: Docked pose of VOA with the PHD2 protein. Docking of VOA with PHD2 was done with Auto dock Tools-4.2.VOA formed H-binding with LEU193,LEU191,HIS282,ARG282,ARG281,ALA190,LEU188, PHE213, GLY213, LEU214, GLU217, ASP278,SER275, SER214, LEU271, THR218 and the binding energy was found to be -7. Figure 1B: Water fall map presentation of ECG/HRV recording of experimental animals In figure, deformities in ECG are pointed by arrows. Groups are G1 -Normal Control saline 2ml/kg); G2 -Toxic (DMBA 8mg/kg i.v); G3-VOA( 1 mg/kg , s.c.); G4)VIN(1mg/kg,i.v.); G5-VOA (0.5mg/kg) + VIN(0.5g/kg,i.v..); G6-VOA (0.5mg/kg) + VIN (0.5g/kg,i.v.); G7-DMSO (3\%,s.c.) Figure 1C: Box cum whisker plot of ECG/HRV recording of experimental animals. 1(Red)-NC (normal saline 2ml/kg,p.o.), 2(Green)-DC (DMSO 3\%, 2ml/kg.p.o.), 3(Blue)-T1 (VOA 1mg/kg,s.c), 4(Light green)-T2 (VIN 1mg/kg,i.v.), 5(Pink)-T3 (VOA+VIN (0.5mg/kg), 6 (Yellow)-T4 (VOA+VIN (1mg/kg), 7 (Grey)-TC (DMBA) 


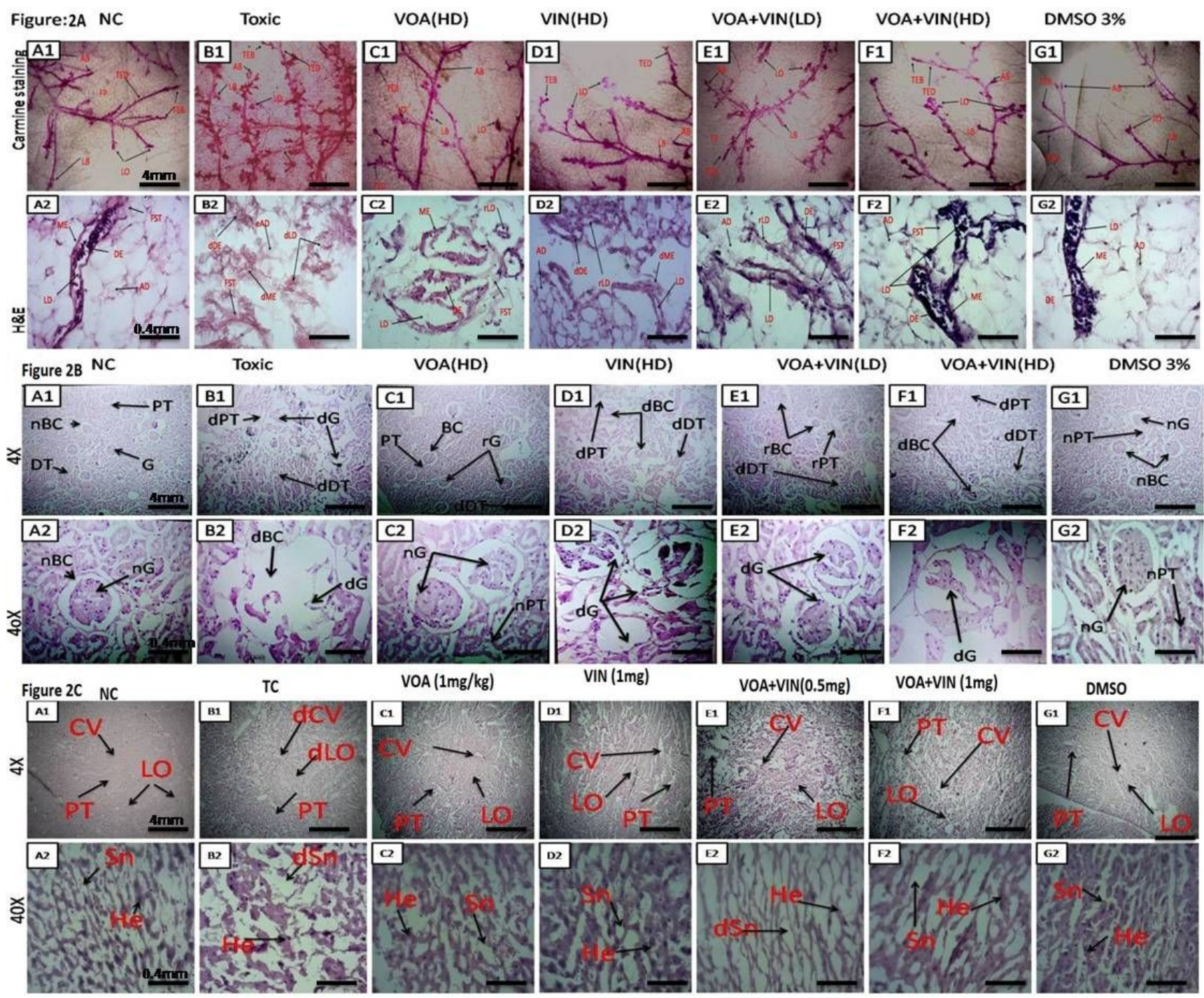

\section{Figure 2}

Figure 2A: Microscopic examination of rat mammary gland tissue through carmine staining (A1-G1) and H\&E staining (A2-G2). A1 and G1 shows that carmine staining of mammary gland tissue of NC and DMSO treated group have lesser number of alveolar buds (AB), lobules (LO), lateral bud (LB) and terminal end duct (TED). B1: DMBA treated TC group showing extensive branching of TED, high number of AB and LB, with large number of LO, C1-F1:Mammary gland of rats treated with monotherapy and combination therapy of VOA $(1 \mathrm{mg} / \mathrm{kg})$ and $\mathrm{VIN}(1 \mathrm{mg} / \mathrm{kg})$ showing lesser number of AB counts, LB,TED and LO when compared to TC. A2 andG2:H\&E staining of mammary gland tissue of NC group showing normal architecture of lactiferous duct (LD), adipose tissue (AT), myoepithelial tissue (ME), ductal epithelium (DE) along with fibrous stromal tissue (FST), B2:H\&E staining of mammary gland tissue of DMBA treated group showing distorted architecture of lactiferous duct (dLD), damaged myoepithelium and damaged ductal epithelium (dME\&dDE), C2-F2:treatment with monotherapy and combination therapy of VOA(1mg/kg) and VIN(1mg/kg) significantly protected further damage to the mammary tissue as evidenced by the restoration of normal architecture LD,AT,DC ME. Figure $2 \mathrm{~B}$ : Effect of VOA and VIN therapy on rat kidney A-G\&a-g: kidney section of rat treated with VOA and VIN at $4 \mathrm{X}$ and $40 \mathrm{X}$ magnifications. A,a-Renal of normal rats showing normal architecture of BC,G,PCT and DCT; B,b-TC rat kidney showing dBC,dG, distorted architecture of PCT and DCT; C,C-VOA treated rat kidney showing normal architecture of BC,G,PCT and DCT; VIN treated rat kidney showing extensive damage to BC,G,PCT and DCT; Ee \& F,f- kideny of rats treated with combination therapy of VOA and VIN showing even more damage to BC,G, and to microtubules(PCT\&DCT); G,gkideny of rat treated 3\%DMSo showing normal architecture of kidney. dBC- damaged bowmans capsule, dG-damaged glomeruls, PCT-proximal convoluted tubule, DCT-distal convoluted tubule Figure 2C: Effect of VOA and VIN therapy on rat Liver A1-G1\&A2-G2-Histologgy of rat treated with VOA nad VIN at 4Xand 40X. A1-A2-liver section of NC rat showing normal architecture of liver lobules(Lo), hepatocutes(He), sinusoids(Sn) and central vein(CV); B1-B2-liver section of TC rats showing dilated central vein, damaged He, distorted Sn and Lo, C1-C2-liver section of VOA treated rat showing little dilation of CV, intact lobular structure, no distortion of Sn and with normal He, D1-D2-liver section of VIN treated rats showing dilated CV, distended portal triads(PT), enhancement in sinusoidal space; E1-E2 and F1-F2-liver section of rat treated with low and high dose combination therapy of VAO and VIN showing extensive distortion of Sn, damage to He, enlargement of central and portal triads and disruption of lobular structure; G1-G2-liver section of rat treated with 3\%DMSo showing normal liver architecture like normal. 
Figure 3A

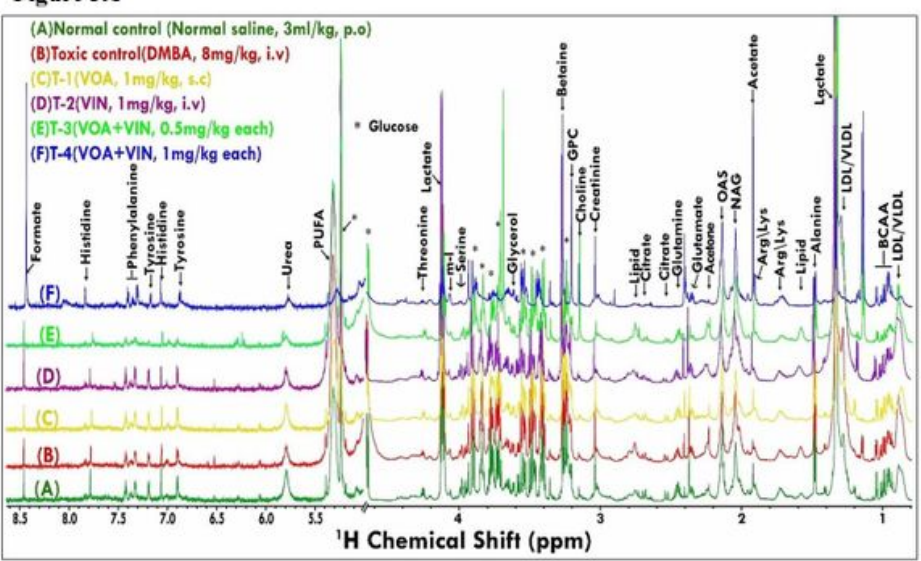

\section{Figure 3B}

Complete data set

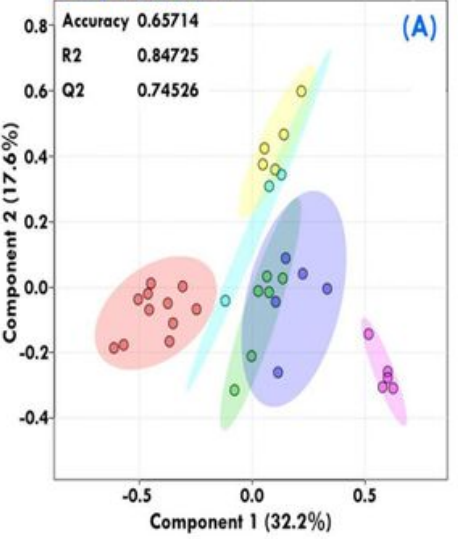

Down-field region from 5.4 to $8.6 \mathrm{ppm}$

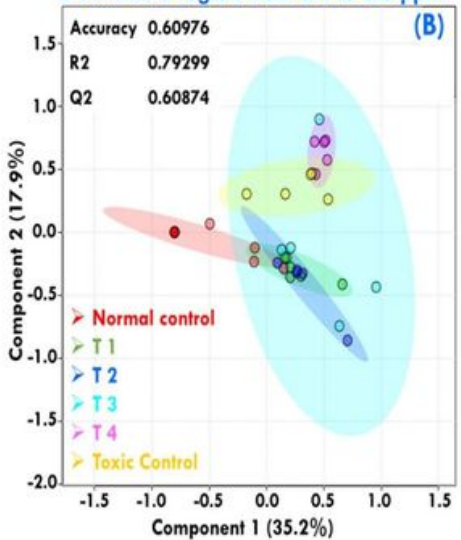

Figure 3C
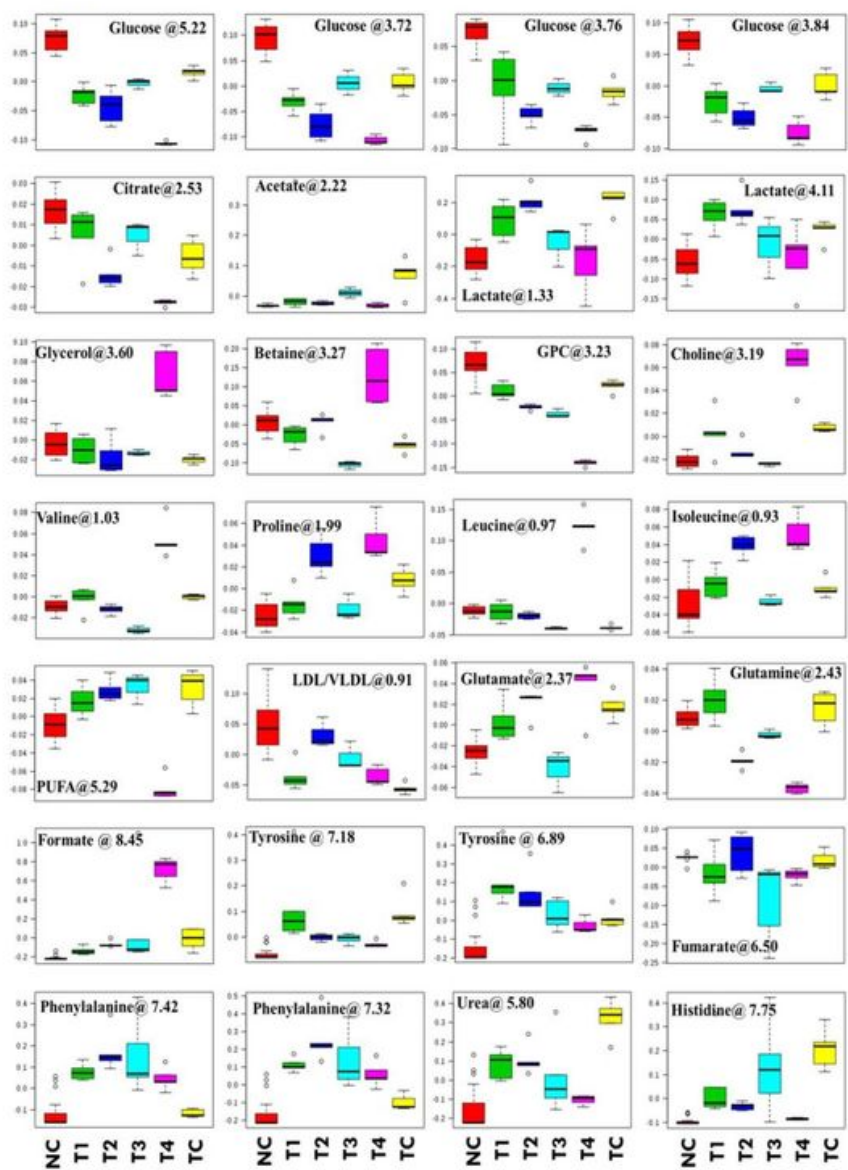

Figure 3

Figure 3A: Stock plot representation of 1D-H-NMRc of serum metabolites of experimental rats: NMR spectra of serum samples are represented in distinguish color formats ( $A, B, C, D, E$, and $F$ shown in above figure). The peaks annotated in the figure below shows the assignments of serum metabolites. The abbreviations used are: LDL/VLDL: Low/very-low density lipoproteins; PUFA: polyunsaturated fatty acids; BCAA: Branch chain amino acids; Leu: Leucine, NAG: N-acetyl glycoproteins; Arg: Arginine; Lys: Lysine; m-I: myo-Inositol; GPC: glycerophosphocholine; and Glucose resonances have been indicated using symbol asterisk “*” Figure 3B: 2D-PLS-DA score plot of rat serum metabolites The combined 2D PLS-DA score plots between the groups clearly depicting that the DMBA induced metabolic changes are largely resetting back to their normal level after Vaocamine and Vincristine treatment in monotherapy and with combine therapy of both drugs as inferred by the decreased separation between treated and normal control groups. In (A), the complete CPMG data matrix is used for PLS-DA modeling; (B) the up-field spectral region (from 5.4 to $8.6 \mathrm{ppm}$, aromatic region) was excluded from the data matrix to evaluate the discriminatory significance of aromatic residues. The 10 fold validation parameters (R2, Q2 and accuracy) for the resulted PLS-DA model are shown in the inset. Figure 3C: Box cum whisker plot of rat serum metabolites Representative box-cum whisker plots showing quantitative variation of relative signal integrals for serum metabolites. For presented metabolite entities, the VIP score $>1$ and statistical significance is at the level of $p \leq 0.05$. In the box plots, the boxes denote the interquartile ranges, horizontal line inside the boxes denote the median and bottom and top boundaries of boxes are 25th and 75th percentiles, respectively. Lowe and upper whiskers are 5th and 95th percentiles, respectively. 
Figure 4
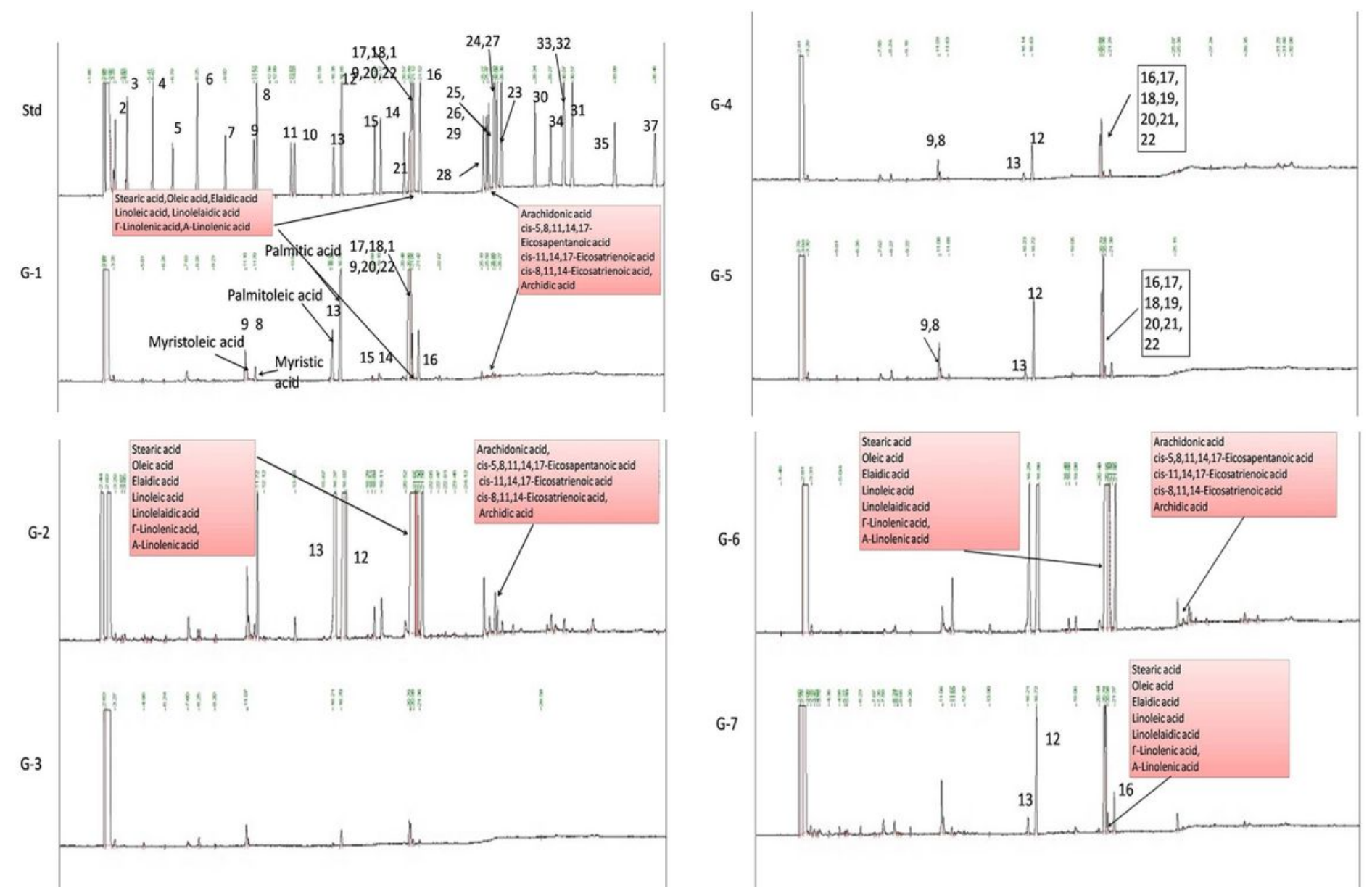

Figure 4

Effect of VOA and VIN on fatty acid composition of mammary gland tissue Std-Standard (Supelco FAME 37component, Sigma Aldrich), G1-Normal control (saline 2ml/kg); G2-Toxic control (DMBA 8mg/kg,i.v.),G3-Voacamine (VOA) (1 mg/kg,s.c.), G4-Vincristine (VIN) (1 mg/kg,i.v.), G5-VOA $(0.5 \mathrm{mg} / \mathrm{kg}, \mathrm{s.c})+$ VIN $(0.5 \mathrm{mg} / \mathrm{kg}$,i.v. $)$, G6-VOA $(1 \mathrm{mg} / \mathrm{kg}$, s.c. $)+$ VIN $(1 \mathrm{mg} / \mathrm{kg}$, i.v.), G7-DC-DMSO $3 \%(2 \mathrm{ml} / \mathrm{kg})]$ 
Figure 5A
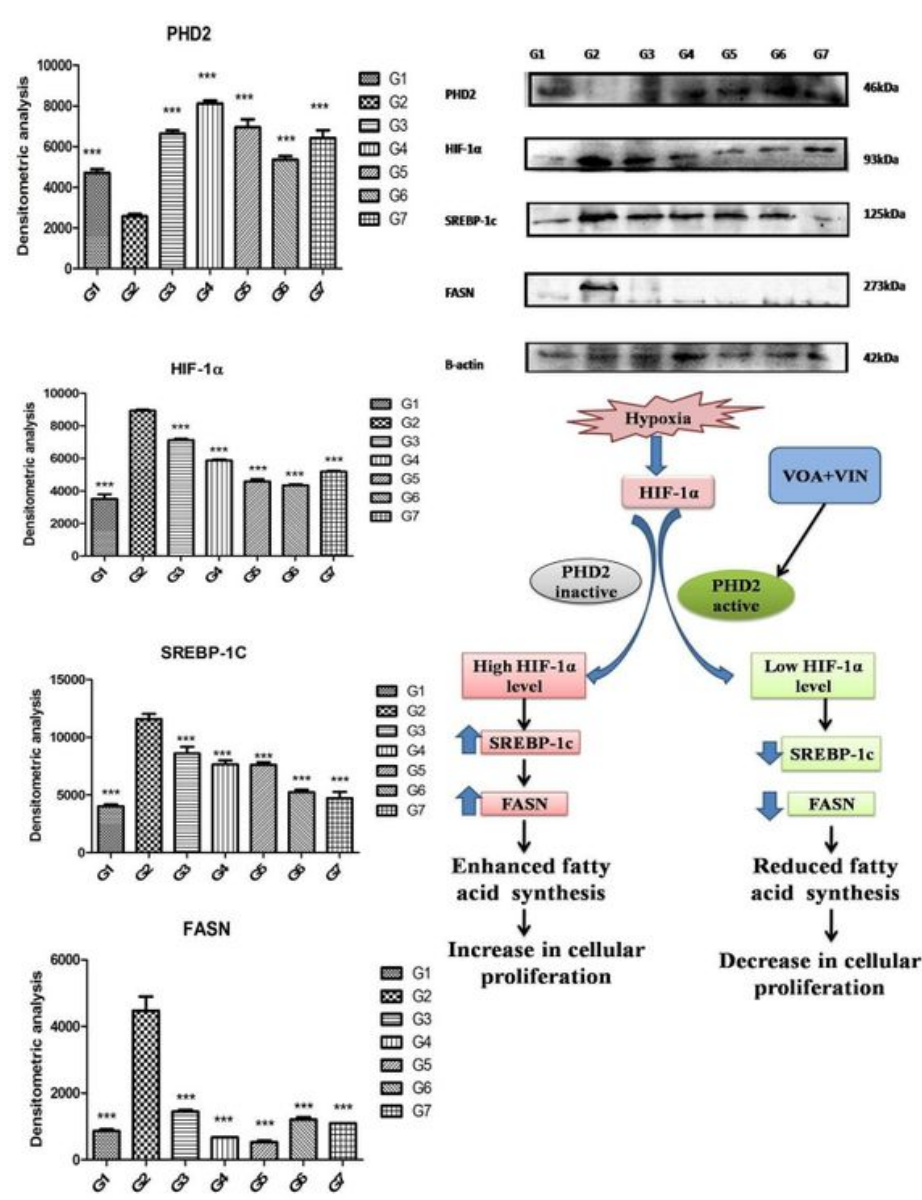

Figure 5B
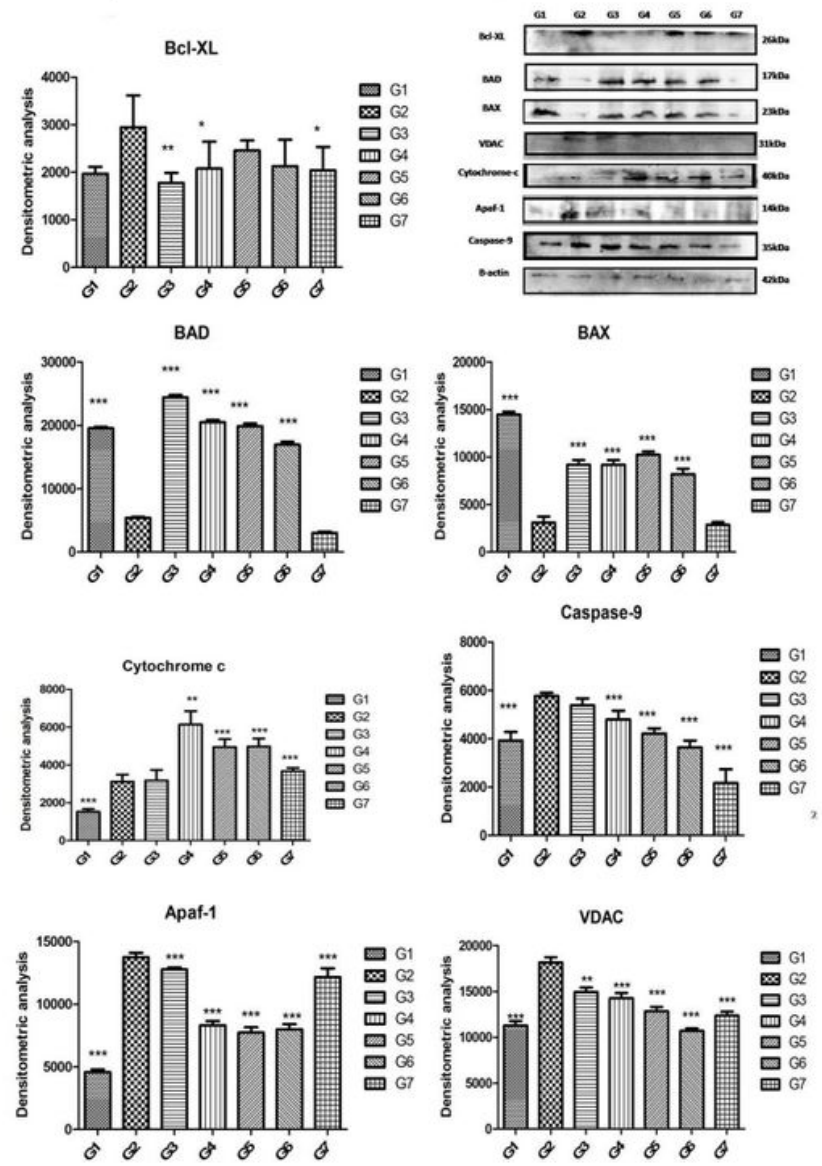

Figure 5

Figure 5A: Effect of VOA and VIN on hypoxic markers and fatty acid synthesis markers. Immunoblotting of respective group [NC-Normal control (saline 2ml/kg); TC-Toxic control (DMBA 8mg/kg,i.v.), T1-Voacamine (VOA) (1 mg/kg,s.c.), T2-Vincristine (VIN) (1mg/kg,i.v.), T3-VOA (0.5mg/kg,s.c) + VIN (0.5mg/kg,i.v.), T4-VOA (1 mg/kg,s.c.) + VIN (1mg/kg, i.v.), DC-DMSO 3\% (2ml/kg)] for HIF-1a, PHD2, SREBP-1c and FASN concluded the hypoxic microenvironment after DMBA administration. Values are presented as mean $+\mathrm{SD}$. Comparisons were made on the basis of one way ANOVA followed by Bonferroni multiple test. All groups were compared to the DMBA treated group $\left({ }^{\star} p<0.05,{ }^{* *} p<0.01,{ }^{* *} p<0.001\right)$. Figure $5 B$ : Effect of VOA and VIN on mitochondrial apoptotic markers. Immunoblotting of respective group [NC-Normal control (saline $2 \mathrm{ml} / \mathrm{kg}$ ); TC-Toxic control (DMBA 8mg/kg,i.v.), T1-Voacamine (VOA) (1 mg/kg,s.c.), T2-Vincristine (VIN) (1 mg/kg,i.v.), T3-VOA (0.5mg/kg,s.c) + VIN (0.5mg/kg,i.v.), T4VOA (1 mg/kg,s.c.) + VIN (1 mg/kg, i.v.), DC-DMSO 3\% (2ml/kg)] for Bcl-XL, BAD, BAX, Cytochrome c, Caspase9, Apaf-1 and VDAC concluded the mitochondrial mediated death apoptsis after DMBA administration. Values are presented as mean + SD. Comparisons were made on the basis of one way ANOVA followed by Bonferroni multiple test. All groups were compared to the DMBA treated group $\left({ }^{\star} p<0.05,{ }^{\star *} p<0.01,{ }^{* \star *} p<0.001\right)$. 
Figure 6

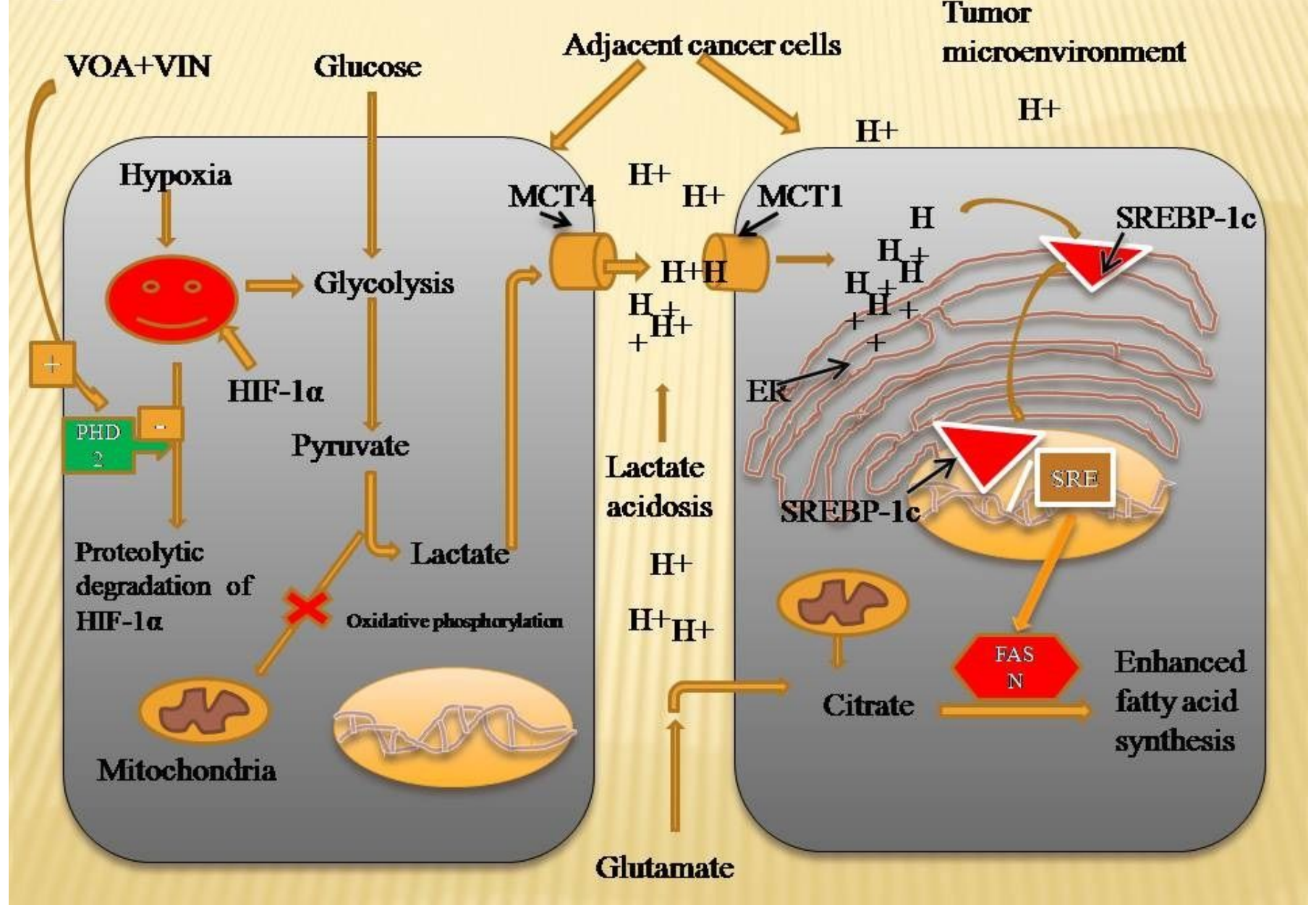

Figure 6

Mechanism of VOA and VIN to inhibit fatty acid synthesis in DMBA induced mammary gland carcinoma of albino wistar rats. Hypoxia activated HIF-1 a enhance lactate acidosis in tumor microenvironment. Dysregulated pH in tumor microenvironment activated SREBP-1 cand FASN expression to speed up fatty synthesis required for plasma membrane synthesis in rapidly proliferating cells. VOA and VIN activated PHD2 enhanced proteolytic degradation of HIF and thus inhibited fatty acid synthesis. HIF-1a hypoxia inducible factor-1a, SREBP-1c-sterolregulatory element binding protein-1c, cFASN: fatty acid synthesis, PHD2-proly hydroxylase2 